Dept. of Math. Univ. of Oslo

Pure Mathematics No. 7

ISSN 0806-2439 APRIL 2006

\title{
CONVERGENCE OF FINITE VOLUME SCHEMES FOR TRIANGULAR SYSTEMS OF CONSERVATION LAWS
}

\author{
K. H. KARLSEN, S. MISHRA, AND N. H. RISEBRO
}

\begin{abstract}
We consider non-strictly hyperbolic systems of conservation laws in triangular form, which arise in applications like three-phase flows in porous media. We device simple and efficient finite volume schemes of Godunov type for these systems that exploit the triangular structure. We prove that the finite volume schemes converge to weak solutions as the discretization parameters tend to zero. Some numerical examples are presented, one of which is related to flows in porous media.
\end{abstract}

\section{Contents}

1. Introduction

2. A triangular three-phase flow model

3. Mathematical Framework

4. Description of numerical schemes

4.1. Staggered Semi-Godunov (SSG) scheme

4.2. Aligned Semi-Godunov (ASG) scheme

5. Convergence Analysis

5.1. The SSG-scheme

5.2. The ASG-scheme

6. Numerical Experiments

References

\section{INTRODUCTION}

We study $2 \times 2$ systems of conservation laws of the form

$$
\begin{cases}u_{t}+f(u)_{x}=0, & (x, t) \in \mathbb{R} \times \mathbb{R}^{+}, \\ v_{t}+g(u, v)_{x}=0, & (x, t) \in \mathbb{R} \times \mathbb{R}^{+}, \\ (u, v)(x, 0)=\left(u_{0}(x), v_{0}(x)\right), & x \in \mathbb{R},\end{cases}
$$

where $u, v$ are the unknowns, whereas the initial values $u_{0}, v_{0}$ and the flux functions $f, g$ are presribed. Triangular systems of conservation laws occur in a variety of models in physics and engineering. As a specific example, we shall discuss a system describing three-phase flow in porous media, see Sections 2 and 6.

Date: April 20, 2006.

2000 Mathematics Subject Classification. 35L65, 35L45, 65M06, 65M12, 76 S05.

Key words and phrases. Conservation laws, systems, triangular form, finite volume schemes, convergence, compensated compactness, flows in porous media.

The research of K. H. Karlsen was supported by an Outstanding Young Investigators Award from the Research Council of Norway. 
System (1.1) is a special case of the followig more general system:

$$
\left\{\begin{array}{c}
\left(u^{1}\right)_{t}+\left(f^{1}\left(u^{1}\right)\right)_{x}=0 \\
\left(u^{2}\right)_{t}+\left(f^{2}\left(u^{1}, u^{2}\right)\right)_{x}=0 \\
\vdots \vdots \\
\left(u^{n}\right)_{t}+\left(f^{n}\left(u^{1}, u^{2}, \ldots, u^{n}\right)\right)_{x}=0
\end{array}\right.
$$

This system is called triangular due to its special structure where evolution of $u_{i}$ does not depend on the succeeding unknowns $\left(u_{i+1}, \ldots, u_{n}\right)$.

Using vector notation, (1.1) reads

$$
U_{t}+F(U)_{x}=0 \quad(x, t) \in \mathbb{R} \times \mathbb{R}^{+},
$$

with $U=(u, v)$ and $F(U)=(f, g)$. The Jacobian matrix for (1.1) is

$$
A=\partial F=\left(\begin{array}{cc}
f_{u} & 0 \\
g_{u} & g_{v}
\end{array}\right) .
$$

The eigenvalues of the above matrix are $\lambda_{1}=f_{u}$ and $\lambda_{2}=g_{v}$. From this it follows that the system (1.1) is hyperbolic but may not be strictly hyperbolic as the wave speeds can coincide. Thus, the key difficulty in analyzing a triangular system like (1.1) is due to this non-strict hyperbolicity or resonance.

Although $2 \times 2$ systems are well studied and several rigorous results have been obtained for them, see [19] and the references therein, none of these results can be directly applied to the system (1.1) on the account of its resonant wave structure. Hence, a different approach exploiting the triangular structure of (1.1) is required in order to analyze the system. This paper in an attempt in this direction. Indeed, our aim is to design numerical schemes of finite volume type for computing approximate solutions of (1.1), and to show that these schemes converge to a weak solution of (1.1), thereby proving existence of weak solutions as well.

If we write (1.1) as a system of equations, we can group different (first order accurate) numerical methods into three main categories: (1) Central type schemes like the Lax-Friedrichs or the Local Lax-Friedrichs (Rusanov) schemes, which do not rely on Riemann solvers. (2) Upwind type schemes like the Godunov and Roe schemes, which are based on (approximate) Riemann solvers. (3) Front tracking schemes, which are based on exact Riemann solvers. Exact Riemann solvers may be difficult to design on the account of a complicated wave structure in the solutions of (1.1). If $f \equiv 0$, then front tracking schemes are viable, see, e.g., [24]. For the triangular system (with degenerate diffusion), see [20] for numerical results with a front tracking based operator splitting method. For a comparison of central and upwind type schemes, see [12].

Numerical schemes based on exact or approximate Riemann solvers are difficult to design and analyze due to the complicated structure of the solutions to (1.1), which is a manifestation of the non-strictly hyperbolic (resonance) feature of the system. Because of this, we propose to use a class of "simple" upwind schemes that exploit the triangular structure of the system (1.1). These schemes are based on the close relationship between (1.1) and scalar conservation laws with discontinuous coefficients, i.e., equations of the form

$$
\begin{cases}w_{t}+h(k(x, t), w)_{x}=0, & (x, t) \in \mathbb{R} \times \mathbb{R}^{+}, \\ w(x, 0)=w_{0}(x), & x \in \mathbb{R},\end{cases}
$$


where $w$ is the conserved variable and the coefficient $k$ is allowed to be discontinuous along curves in the $(x, t)$ plane. Indeed, one can see that since the evolution of $u$ is independent of $v$ in (1.1), we can evolve $u$ and treat it is a coefficient in the evolution equation for $v$, thus reducing (1.1) to an equation of the form (1.3). In this paper, we are going to exploit this close relationship to device Godunov type numerical schemes. These schemes are based on Riemann solvers for a corresponding conservation law with discontinuous flux (1.3).

Conservation laws with discontinuous coefficients occur in a wide variety of models, such as in two phase flows in heterogeneous porous media (see, e.g., [17]), in models for sedimentation of suspensions in a clarifier-thickener unit (see, e.g., [8]), and in models for traffic flow (see, e.g., [5]). These equations have been studied in several papers. An incomplete list includes $[1,2,3,6,13,17,24,21,22,23$, $28,26,27,29,32,33]$ (see the references therein for a more complete picture). A key difficulty of the analysis is the nonlinear resonant behavior, which means that one cannot expect to bound the total variation of the conserved quantities directly but only when measured under a certain singular mapping. The singular mapping method, used in most of the literature on these problems, is however difficult to apply if the coefficient $k$ in (1.3) is discontinuous both in space and time and additionally is merely $B V$ regular, i.e., not necessarily piecewise smooth. Herein we will therefore take a different approach avoiding the singular mapping altogether.

The schemes we shall analyze are proposed in Section 4 and we shall refer to them as Semi-Godunov schemes. These schemes are very easy to implement as they do not rely on the full wave structure of the $2 \times 2$ system. Their numerical dissipation is much smaller than that of the Lax-Friedrichs scheme, and their numerical performance is comparable with front tracking schemes. Additionally, we show that these schemes converge to a weak solution of (1.1). For the convergence analysis, since $B V$ estimates are not available and the singular mapping approach seems difficult to implement for our system, we employ the Murat-Tartar compensated compactness method $[30,31]$. Parts of the $W_{\text {loc }}^{-1,2}$ analysis relies heavily on the particular structure of our system (1.1).

The remainder of this paper is organised as follows: In Section 2 we describe a reduced three-phase flow model where (1.1) arises. The mathematical framework and detailed assumptions on the initial data and the fluxes are described in Section 3. In Section 4 we describe the numerical schemes for (1.1). Convergence analyses of the numerical schemes are carried out in Section 5. Some numerical results are presented in section 6 .

\section{A tRiAngular THREE-PHASE FLOW MODEL}

Simulation of a variety of oil recovery processes involve models of three-phase flow in porous media. Often the three-phases of interest are oil, gas, and water. As a model we consider incompressible, immiscible three-phase flow in a one-dimensional homogeneous and isotropic reservoir (see, e.g., [9]). The oil, water, and gas saturations are given by $S_{o}, S_{w}, S_{g}$ respectively.

The mass conservation equation for phase $l=w, o, g$ reads

$$
\phi\left(S_{l}\right)_{t}+\left(U_{l}\right)_{x}=0
$$


where $\phi$ is the porosity of the medium and $U_{l}$ is the Darcy velocity or flow rate corresponding to each phase $l$. By Darcy's law, the flow rate is given by

$$
U_{l}=-k \lambda_{l}\left(\frac{\partial P_{l}}{\partial x}-\rho_{l} g\right) \quad l=w, o, g,
$$

where $k$ denotes the absolute permeability of the medium, $\lambda_{l}$ the mobility (relative permeability divided by viscosity), $P_{l}, \rho_{l}$ the density of phase $l$, and $g$ the gravitational constant. We assume that the flow is incompressible i.e., the total flow rate $q=\sum_{l=w, o, g} U_{l}$ is a constant. For the sake of simplicity, we assume that the differences in the capillary pressures between the phases are zero. This assumption is reasonable when the total flow rate is high (the flow is convection dominated).

By adding the mass conservation equations (2.1) and using the above assumptions, we arrive at the following $2 \times 2$ system of conservation laws:

$$
\left\{\begin{array}{l}
\phi\left(S_{g}\right)_{t}+\left(F_{g}\left(S_{g}, S_{w}, S_{o}\right)\right)_{x}=0, \\
\phi\left(S_{w}\right)_{t}+\left(F_{w}\left(S_{g}, S_{w}, S_{o}\right)\right)_{x}=0, \\
S_{g}+S_{w}+S_{o}=1,
\end{array}\right.
$$

where the flux functions are given by,

$$
\begin{gathered}
F_{g}\left(S_{g}, S_{w}, S_{o}\right)=\frac{q \lambda_{g}}{\lambda_{t}}+\frac{k}{\lambda_{t}} \lambda_{w} \lambda_{g}\left(g_{w}-g_{g}\right)+\frac{k}{\lambda_{t}} \lambda_{o} \lambda_{g}\left(g_{o}-g_{g}\right), \\
F_{w}\left(S_{g}, S_{w}, S_{o}\right)=\frac{q \lambda_{w}}{\lambda_{t}}+\frac{k}{\lambda_{t}} \lambda_{w} \lambda_{g}\left(g_{g}-g_{w}\right)+\frac{k}{\lambda_{t}} \lambda_{o} \lambda_{w}\left(g_{o}-g_{w}\right),
\end{gathered}
$$

where $\lambda_{t}=\lambda_{o}+\lambda_{g}+\lambda_{w}$ is the total mobility and $g_{l}=\rho_{l} g$.

It is well known that (2.2) can be a system of mixed type, i.e., there may exist elliptic regions where the eigenvalues are imaginary. It is outside the scope of this paper to discuss this. Instead we refer to [7] (and the references therein) for a review some of the current views that exist today regarding mathematical and numerical theory for mixed type systems.

In many situations the mobility of the gas phase is much larger than that of the other phases. This means that the flux of gas is largely independent of whether the other phase is oil or water. As a consequence

$$
F_{g}\left(S_{g}, S_{w}, S_{o}\right)=\tilde{F}\left(S_{g}, 1-S_{g}\right)=\hat{F}\left(S_{g}\right) .
$$

Assuming this relationship, system (2.2) reduces to the following system

$$
\begin{aligned}
\left(S_{g}\right)_{t}+\left(\hat{F}_{g}\left(S_{g}\right)\right)_{x} & =0 \\
\left(S_{w}\right)_{t}+\left(F_{w}\left(S_{g}, S_{w}\right)\right)_{x} & =0,
\end{aligned}
$$

where we have set the porosity to unity. The above equation is a special case of (1.1). We refer to [20] for the model when capillary forces are included.

It is to be emphasized that that although the assumption of independence of the gas phase is not valid for all fractional flow functions, there exists a large class of fractional flow functions for which this assumption appears to be reasonable. In view of the fact that this assumption makes the model simpler and much more tractable, we can use this "reduced" model in several situations. A careful numerical study of this model (2.3) as an approximation to the full three-phase flow model needs to be carried out. An essential ingredient for this program is the development of efficient numerical schemes for (1.1). 
We remark that a one dimensional model like the one that we are using is a good starting point for developing numerical schemes for the full three dimensional model where one can use the one dimensional numerical fluxes in directions normal to volume interfaces or along streamlines.

\section{Mathematical Framework}

We start by stating precise conditions on the flux functions $f, g$. Fix real numbers $s_{0}, S_{0}, \alpha, \beta$ such that $s_{0} \leq S_{0}$ and $\alpha \leq \beta$. Then we assume that $f$ and $g$ satisfy

A.1 $f \in \operatorname{Lip}([\alpha, \beta])$,

A.2 $g\left(u_{1}, s_{0}\right)=g\left(u_{2}, s_{0}\right), g\left(u_{1}, S_{0}\right)=g\left(u_{2}, S_{0}\right)$ for all $u_{1}, u_{2} \in[\alpha, \beta]$,

A.3 $u \mapsto g(u, v) \in C^{1}([\alpha, \beta])$ for each $v \in\left[s_{0}, S_{0}\right]$,

A.4 $v \mapsto g(u, v) \in C^{2}\left(\left[s_{0}, S_{0}\right]\right)$ for each $u \in[\alpha, \beta]$,

A.5 $u \mapsto g_{v v}(u, v) \in \operatorname{Lip}([\alpha, \beta])$ for each $v \in\left[s_{0}, S_{0}\right]$.

A.6 $v \mapsto g(u, v)$ is genuinely nonlinear, for each $u \in[\alpha, \beta]$, that is,

$$
g_{v v}(u, v) \neq 0 \text { for a.e. } v \in\left[s_{0}, S_{0}\right] \text {, for each } u \in[\alpha, \beta] \text {. }
$$

Conditions A.1, A.4, and A.6 are the usual smoothness assumptions on the fluxes. Condition A.2 is a sufficient condition to obtain $L^{\infty}$ bounds on $v$ and can be relaxed in several ways. We have chosen to use this form since it holds for the three-phase flow model (2.3). Condition A.6 is required to achieve strong convergence of the approximate solutions with the compensated compactness method. Condition A.5 is a technical condition used in the $W_{\text {loc }}^{-1,2}$ compactness analysis of the Aligned SemiGodunov scheme defined in Subsection 4.2. All the assumptions are quite general. In particular, we require no further assumptions on the shape of the fluxes.

Regarding the initial data we assume the following conditions:

A.7 $u_{0} \in L^{\infty}(\mathbb{R})$ with $\alpha \leq u_{0} \leq \beta$ for a.e $x \in \mathbb{R}$,

A. $8 u_{0} \in B V(\mathbb{R})$,

A.9 $s_{0} \leq v_{0}(x) \leq S_{0}$ for all $x \in \mathbb{R}$.

A weak solution to $(1.2)$ is a pair of functions $U=(u, v)$ such that

$$
\int_{\mathbb{R}^{+}} \int_{\mathbb{R}}\left(U \varphi_{t}+F(U) \varphi_{x}\right) d x d t+\int_{\mathbb{R}} U(x, 0) \varphi(x, 0) d x=0, \quad \forall \varphi \in C_{c}^{\infty}\left(\mathbb{R} \times \mathbb{R}^{+}\right) .
$$

It is well known that weak solutions of conservation laws are not unique, and therefore the solution concept has to be supplemented with additional admissibility criteria, so called entropy conditions. We refer to [19] for an introduction to entropy conditions and the general theory of conservation laws.

A pair of functions $(\eta(u, v), Q(u, v))$, where $u$ is considered as a fixed parameter in $[\alpha, \beta]$, is called a scalar entropy/entropy flux pair for the equation $v_{t}+g(u, v)_{x}=0$ if $v \mapsto \eta(u, v)$ is $C^{2}$ regular and

$$
Q_{v}(u, v)=\eta_{v}(u, v) g_{v}(u, v), \quad \text { for all } v \in\left[s_{0}, S_{0}\right] .
$$

Suitably modified for our purposes, the compensated compactness lemma used in [23] reads as follows:

Lemma 3.1. Let $u$ be the unique entropy solution $u$ of the single conservation law

$$
u_{t}+f(u)_{x}=0, \quad u(x, 0)=u_{0}(x) \in L^{\infty}(\mathbb{R}) \cap B V(\mathbb{R}),
$$

and let $\left\{v^{\varepsilon}\right\}_{\varepsilon>0}$ be a sequence of functions on $\mathbb{R} \times \mathbb{R}^{+}$such that for all $\varepsilon>0$

(i) $s_{0} \leq v^{\varepsilon} \leq S_{0}$, and 
(ii) the sequences

$$
\left\{\eta_{1}\left(v^{\varepsilon}\right)_{t}+Q_{1}\left(u, v^{\varepsilon}\right)_{x}\right\}_{\varepsilon>0}, \quad\left\{\eta_{2}\left(u, v^{\varepsilon}\right)_{t}+Q_{2}\left(u, v^{\varepsilon}\right)_{x}\right\}_{\varepsilon>0}
$$

belong to a compact subset of $W_{\mathrm{loc}}^{-1,2}\left(\mathbb{R} \times \mathbb{R}^{+}\right)$, where for all $u, v$

$$
\begin{gathered}
\eta_{1}(v)=v-c, \quad Q_{1}(u, v)=g(u, v)-g(u, c) \\
\eta_{2}(u, v)=g(u, v)-g(u, c), \quad Q_{2}(u, v)=\int_{c}^{v}\left(g_{v}(u, \xi)\right)^{2} d \xi
\end{gathered}
$$

for all $c \in \mathbb{R}$.

Then there exists a subsequence of $\left\{v^{\varepsilon}\right\}_{\varepsilon>0}$ that converges in $L_{\mathrm{loc}}^{p}\left(\mathbb{R} \times \mathbb{R}^{+}\right) \forall p<\infty$ and a.e. to a bounded function $v$.

We also need the following technical result (see, e.g., [10, 25]):

Lemma 3.2. Let $\Omega \subset \mathbb{R}^{d}$ be a bounded open set and $q, p, r$ numbers such that $1<q \leq p<r<\infty$, then

$$
\begin{aligned}
& \text { (compact set of } \left.W^{-1, q}(\Omega)\right) \cap\left(\text { bounded set of } W^{-1, r}(\Omega)\right) \\
& \subset\left(\text { compact set of } W^{-1, p}(\Omega)\right) .
\end{aligned}
$$

\section{Description of NUMERICAL SCHEMES}

In this section we describe the Semi-Godunov type schemes for (1.1). Let $\Delta t$ and $\Delta x$ denote the time step and mesh size respectively. For simplicity we use a uniform mesh in both space and time, although variable step sizes can be handled in the same manner. We assume that the time step and the mesh size satisfy the following CFL condition:

$$
\lambda M \leq \frac{1}{2}, \quad \lambda=\frac{\Delta t}{\Delta x}, \quad M=\max \left\{\max _{u \in[\alpha, \beta]}\left|f_{u}\right|, \max _{u \in[\alpha, \beta], v \in\left[s_{0}, S_{0}\right]}\left|g_{v}\right|\right\},
$$

Let $t^{n}=n \Delta t$, and $x_{j}=j \Delta x$ for integers $n=0,1,2, \ldots$ and $j=\ldots,-3 / 2,-1$, $-1 / 2,0,1 / 2,1,3 / 2,2, \ldots$. Let $I_{j}$ and $I^{n}$ denote the intervals

$$
I_{j}=\left[x_{j-1 / 2}, x_{j+1 / 2}\right), \quad I^{n}=\left[t^{n}, t^{n+1}\right) .
$$

Set

$$
\chi_{j}^{n}(x, t)=\chi_{I_{j}}(x) \chi_{I^{n}}(t),
$$

where $\chi_{\Omega}$ denotes the characteristic function of a set $\Omega$.

4.1. Staggered Semi-Godunov (SSG) scheme. For this scheme, we are going to stagger the discretization of the two unknowns $u$ and $v$.

Define $U_{j}^{0}=\left(u_{j+1 / 2}^{0}, v_{j}^{0}\right)$ as

$$
u_{j+1 / 2}^{0}=\frac{1}{\Delta x} \int_{x_{j}}^{x_{j+1}} u_{0}(x) d x, \quad v_{j}^{0}=\frac{1}{\Delta x} \int_{x_{j-1 / 2}}^{x_{j+1 / 2}} v_{0}(x) d x .
$$

Given given $U_{j}^{n}=\left(u_{j+1 / 2}^{n}, v_{j}^{n}\right)$, we shall determine $U_{j}^{n+1}=\left(u_{j+1 / 2}^{n+1}, v_{j}^{n+1}\right)$. We start by updating $u_{j+1 / 2}^{n}$. This is straightforward as the evolution of $u$ does not depend on the unknown $v$. We use the standard Godunov scheme, see [18]:

$$
u_{j+1 / 2}^{n+1}=u_{j+1 / 2}^{n}-\lambda\left(f^{\mathrm{G}}\left(u_{j+1 / 2}^{n}, u_{j+3 / 2}^{n}\right)-f^{\mathrm{G}}\left(u_{j-1 / 2}^{n}, u_{j+1 / 2}^{n}\right)\right),
$$


where $f^{\mathrm{G}}$ is the standard scalar Godunov numerical flux:

$$
f^{\mathrm{G}}(a, b)= \begin{cases}\min _{\theta \in[a, b]} f(\theta), & \text { if } a \leq b \\ \max _{\theta \in[b, a]} f(\theta), & \text { otherwise. }\end{cases}
$$

We define an approximate solution $u^{\Delta x}$ on $\mathbb{R} \times \mathbb{R}^{+}$by

$$
u^{\Delta x}(x, t)=\sum_{n, j+1 / 2} \chi_{j+1 / 2}^{n}(x, t) u_{j+1 / 2}^{n} .
$$

Next, we are going to use the function $u^{\Delta}$ to define a Riemann solver for $v$. At any time level $t^{n}$, we will substitute $u^{\Delta x}\left(x, t^{n}\right)$ instead of $u$ in the conservation law for $v$. More precisely, for $(x, t) \in I_{j+1 / 2} \times I^{n}$ we define $v^{\Delta x}$ to be the solution of the following conservation law:

$$
v_{t}^{\Delta x}+g\left(u_{j+1 / 2}^{n}, v^{\Delta x}\right)_{x}=0, \quad v^{\Delta x}\left(x, t^{n}\right)= \begin{cases}v_{j}^{n}, & x<x_{j+1 / 2}, \\ v_{j+1}^{n}, & x>x_{j+1 / 2} .\end{cases}
$$

Since waves from different Riemann problems at $t^{n}$ do not interact by the CFLcondition, we can use a Godunov scheme to determine $v_{j}^{n+1}$. We evolve the solution of the Riemann problem until $t=t^{n+1}$. At time $t=t^{n+1}$, we define $v_{j}^{n+1}$ by averaging over grid cells $I_{j}$ :

$$
v_{j}^{n+1}=\frac{1}{\Delta x} \int_{x_{j-1 / 2}}^{x_{j+1 / 2}} v^{\Delta x}\left(x, t^{n+1}-\right) d x .
$$

This gives the formula,

$$
v_{j}^{n+1}=v_{j}^{n}-\lambda\left(g^{\mathrm{G}}\left(u_{j+1 / 2}^{n}, v_{j}^{n}, v_{j+1}^{n}\right)-g^{\mathrm{G}}\left(u_{j-1 / 2}^{n}, v_{j-1}^{n}, v_{j}^{n}\right)\right),
$$

where $g^{\mathrm{G}}(u, a, b)$ is the standard Godunov flux (4.2) corresponding to the flux function $v \mapsto g(u, v)$. Collecting the updates for $u$ and $v$, we get the following finite volume scheme:

$$
\begin{gathered}
u_{j+1 / 2}^{n+1}=u_{j+1 / 2}^{n}-\lambda\left(f^{\mathrm{G}}\left(u_{j+1 / 2}^{n}, u_{j+3 / 2}^{n}\right)-f^{\mathrm{G}}\left(w_{j-1 / 2}^{n}, u_{j+1 / 2}^{n}\right)\right), \\
v_{j}^{n+1}=v_{j}^{n}-\lambda\left(g^{\mathrm{G}}\left(u_{j+1 / 2}^{n}, v_{j}^{n}, v_{j+1}^{n}\right)-g^{\mathrm{G}}\left(u_{j-1 / 2}^{n}, v_{j-1}^{n}, v_{j}^{n}\right)\right) .
\end{gathered}
$$

We coin (4.6) the Staggered Semi-Godunov (SSG) scheme due to the staggered discretizations of the coefficient and the unknown. This scheme is similar in spirit to the schemes in $[32,33,21]$ for conservation laws with discontinuous coefficients.

For the purpose of analysis, we define an approximate solution $U^{\Delta x}=\left(u^{\Delta x}, v^{\Delta x}\right)$ on $\mathbb{R} \times \mathbb{R}^{+}$by (4.3) and (4.4), (4.5).

4.2. Aligned Semi-Godunov (ASG) scheme. Unlike the SSG-scheme (4.6), for the ASG-scheme we align the discretizations of both the unknowns. As a result the ASG-scheme becomes more complicated to implement and analyze than the SSG-scheme, but we have found that it gives slightly better results.

Define $U_{j}^{0}=\left(u_{j}^{0}, v_{j}^{0}\right)$ by

$$
u_{j}^{0}=\frac{1}{\Delta x} \int_{x_{j-1 / 2}}^{x_{j+1 / 2}} u_{0}(x) d x, \quad v_{j}^{0}=\frac{1}{\Delta x} \int_{x_{j-1 / 2}}^{x_{j+1 / 2}} v_{0}(x) d x .
$$


As in the SSG-scheme, we first update for $u$ using the standard Godunov scheme:

$$
u_{j}^{n+1}=u_{j}^{n}-\lambda\left(f^{\mathrm{G}}\left(u_{j}^{n}, u_{j+1}^{n}\right)-f^{\mathrm{G}}\left(u_{j-1}^{n}, u_{j}^{n}\right)\right),
$$

where the numerical flux $f^{\mathrm{G}}$ is defined in (4.2). Equipped with (4.1), we define an approximate solution $u^{\Delta x}$ on $\mathbb{R} \times \mathbb{R}^{+}$by

$$
u^{\Delta x}(x, t)=\sum_{n, j} \chi_{j}^{n}(x, t) u_{j}^{n} .
$$

As in the SSG-scheme, we use $u^{\Delta x}(x, t)$ to define the evolution of $v^{\Delta x}$. More precisely, we define $v^{\Delta x}$ for $(x, t) \in I_{j+1 / 2} \times I^{n}$ by solving the following local Riemann problem at the interface $x_{j+1 / 2}$ :

$$
\begin{aligned}
& v_{t}^{\Delta x}+g\left(u_{j}^{n}, v^{\Delta x}\right)_{x}=0, \quad \text { if } x<x_{j+1 / 2}, \\
& v_{t}^{\Delta x}+g\left(u_{j+1}^{n}, v^{\Delta x}\right)_{x}=0, \quad \text { if } x>x_{j+1 / 2}, \\
& v^{\Delta x}\left(x, t^{n}\right)= \begin{cases}v_{j}^{n}, & x<x_{j+1 / 2}, \\
v_{j+1}^{n}, & x>x_{j+1 / 2} .\end{cases}
\end{aligned}
$$

As we have aligned the discretization of both the unknowns, we end up with a Riemann problem corresponding to a single conservation law with a discontinuous coefficient. The Riemann problem (4.8) can be solved (see, e.g., [13, 16, 17]), and an explicit formula for the (Godunov type) numerical flux has been obtained in $[3,4,27]$ for a large class of flux functions.

We define $v_{j}^{n+1}$ by averaging, cf. (4.5), and obtain

$$
v_{j}^{n+1}=v_{j}^{n}-\lambda\left(g_{\mathrm{A}}^{\mathrm{R}}\left(\left(u_{j}^{n}, u_{j+1}^{n}\right),\left(v_{j}^{n}, v_{j+1}^{n}\right)\right)-g_{\mathrm{A}}^{\mathrm{R}}\left(\left(u_{j-1}^{n}, u_{j}^{n}\right),\left(v_{j-1}^{n}, v_{j}^{n}\right)\right)\right),
$$

where $g_{\mathrm{A}}^{\mathrm{R}}(k, l)(a, b)$ is the Godunov numerical flux corresponding to the Riemann problem with left flux function $g(k,$.$) , right flux function g(l,$.$) and Riemann data$ $a$ (left) and $b$ (right). As mentioned above, explicit formulas for $g_{\mathrm{A}}^{\mathrm{R}}$ can be given in many cases. For example, if $v \mapsto g(u, v)$ has at most one minimum and no maxima for every $u$, then the explicit formula is

$$
g_{\mathrm{A}}^{\mathrm{R}}((k, l),(a, b))=\max \left\{g\left(k, \max \left(a, \theta_{k}\right)\right), g\left(l, \min \left(\theta_{l}, b\right)\right\},\right.
$$

where $\theta_{k}, \theta_{l}$ are the minimum points of $g(k, \cdot)$ and $g(l, \cdot)$ respectively. Explicit formulas in other (non-convex) cases are given in [4, 27].

Finally, we define an approximate solution $U^{\Delta x}=\left(u^{\Delta x}, v^{\Delta x}\right)$ on $\mathbb{R} \times \mathbb{R}^{+}$via formulas (4.7) and (4.8).

\section{Convergence Analysis}

In this section we prove that the approximate solutions generated by the SSGscheme (4.6) and the ASG-scheme (4.9) converge to weak solutions of (1.1). We start by analyzing the SSG-scheme (4.6) and then detail the differences for the ASG-scheme. In what follows and without loss of generality we will assume that the approximate solutions (and their initial data) have compact support, that is, there exist constants $X, T>0$ independent of $\Delta x$ such that

$$
\operatorname{supp}\left(u^{\Delta x}\right), \operatorname{supp}\left(v^{\Delta x}\right) \subset[-X, X] \times[0, T] .
$$


5.1. The SSG-scheme. We will carry out the above steps for the SSG-scheme (4.6). Using standard theory for scalar conservation laws, it is straightforward to establish the following facts:

Lemma 5.1. Let $u_{j+1 / 2}^{n}, u^{\Delta x}$ be defined by (4.1) and (4.3). Then

$$
\begin{gathered}
\alpha \leq u_{j+1 / 2}^{n} \leq \beta, \quad \text { for } j \in \mathbb{Z}, n=0,1,2, \ldots, \\
\sum_{j}\left|u_{j+1 / 2}^{n+1}-u_{j+1 / 2}^{n}\right| \leq \sum_{j}\left|u_{j+1 / 2}^{n}-u_{j+1 / 2}^{n-1}\right|, \quad n \geq 1, \\
\sum_{j}\left|u_{j+1 / 2}^{n}-u_{j-1 / 2}^{n}\right| \leq \sum_{j}\left|u_{j+1 / 2}^{n-1}-u_{j-1 / 2}^{n-1}\right|, \quad n \geq 1 .
\end{gathered}
$$

Furthermore, the sequence $\left\{u^{\Delta x}\right\}_{\Delta x>0}$ converges to the unique entropy solution $u \in L^{\infty}\left(\mathbb{R} \times \mathbb{R}^{+}\right) \cap B V\left(\mathbb{R} \times \mathbb{R}^{+}\right)$of the first equation in (1.1). The convergence takes place in $\left.L_{\mathrm{loc}}^{p}\left(\mathbb{R} \times \mathbb{R}^{+}\right)\right) \forall p<\infty$ and a.e. in $\mathbb{R} \times \mathbb{R}^{+}$.

The boundedness of $v^{\Delta x}$ is a standard result, and follows from Assumption A.2 and the monotonicity properties of $g^{\mathrm{G}}$, see [21]. We state the result in a lemma.

Lemma 5.2. Let $v_{j}^{n}$ be generated by the SSG-scheme (cf. Subsection 4.1), and

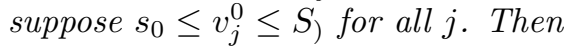

$$
s_{0} \leq v_{j}^{n} \leq S_{0} \text { for all } j \text { and } n \geq 0 .
$$

We remark that our particular $L^{\infty}$ bound holds under Assumption A.2, but one can show $L^{\infty}$ bounds under more general conditions, see [33].

The next step in the convergence analysis is to provide an entropy dissipation estimate for the SSG-scheme (4.6). A entropy estimate for the Lax-Friedrichs scheme for (1.3) was proved in [23]. The approach of [23] is based on cell entropy inequalities and does not extend to three point schemes like the Godunov type scheme based on Riemann solvers as described in the previous section. Therefore we will employ the original framework of DiPerna [14], of viewing finite volume schemes as layered integral average methods. For other applications based on this approach, see $[15,11]$ and the references therein.

Here and in what follows, we use the notation

$$
\llbracket A \rrbracket(x, t)=\lim _{h \rightarrow 0} A(x-h, t)-A(x+h, t),
$$

for any quantity $A=A(x, t)$.

Let $(\eta, Q)$ be a scalar entropy/entropy flux pair. Pick a test function $\varphi$ having compact support in $\mathbb{R} \times[0, T]$ with $T=N \Delta t$ for some integer $N$. By the Gauss-Green formula and the local structure of the approximations $v^{\Delta x}\left(v^{\Delta x}\right.$ is the solution of a Riemann problem at each interface $x_{j+1 / 2}$ ),

$$
\begin{gathered}
\iint_{\mathbb{R} \times \mathbb{R}^{+}} \eta\left(u^{\Delta x}, v^{\Delta x}\right) \varphi_{t}+Q\left(u^{\Delta x}, v^{\Delta x}\right) \varphi_{x} d x d t \\
=I_{1}(\varphi)+I_{2}(\varphi)+I_{3}(\varphi)+I_{4}(\varphi)
\end{gathered}
$$

where

$$
\begin{aligned}
& I_{1}(\varphi)=\left.\int_{\mathbb{R}} \eta\left(u^{\Delta x}(x, t), v^{\Delta x}(x, t)\right) \varphi(x, t)\right|_{t=0} ^{t=T} d x, \\
& I_{2}(\varphi)=\sum_{n} \int_{\mathbb{R}}\left[\eta\left(u^{\Delta x}\left(x, t^{n}-\right), v^{\Delta x}\left(x, t^{n}-\right)\right)\right.
\end{aligned}
$$




$$
\begin{gathered}
\left.-\eta\left(u^{\Delta x}\left(x, t^{n}+\right), v^{\Delta x}\left(x, t^{n}+\right)\right)\right] \varphi\left(x, t^{n}\right) d x, \\
I_{3}(\varphi)=\sum_{n, j} \sum_{\sigma} \int_{t^{n}}^{t^{n+1}}[(\sigma \llbracket \eta \rrbracket-\llbracket Q \rrbracket) \varphi]\left(x_{j+1 / 2}+\sigma t, t\right) d t, \\
I_{4}(\varphi)=\sum_{n, j} \int_{t^{n}}^{t^{n+1}}\left[Q\left(u_{j-1 / 2}^{n}, v_{j}^{n}\right)-Q\left(u_{j+1 / 2}^{n}, v_{j}^{n}\right)\right] \varphi\left(x_{j}, t\right) d t,
\end{gathered}
$$

and the summation over $\sigma$ extends to all shocks with speed $\sigma$ in the solution of the Riemann problem at the interface $x_{j+1 / 2}$.

Lemma 5.3. Let $v_{j}^{n}, v^{\Delta x}$ be generated by the SSG-scheme (cf. Subsection 4.1). We have

$$
\sum_{j, n} \int_{x_{j-1 / 2}}^{x_{j+1 / 2}}\left|v_{j}^{n}-v^{\Delta x}\left(x, t^{n}-\right)\right|^{2} d x \leq C,
$$

where $C=C(X, T)$ is constant independent of $\Delta x$ and $X, T$ are defined in (5.1). Additionally,

$$
\sum_{n, j} \sum_{\sigma} \int_{t^{n}}^{t^{n+1}} \llbracket \sigma \eta-Q \rrbracket\left(x_{j+1 / 2}+\sigma t, t\right) d t \leq C .
$$

Proof. In this proof we use the convex entropy/entropy flux pair $(\eta, Q)$ defined by $\eta(u, v)=\frac{1}{2} v^{2}, Q_{v}(u, v)=v g_{v}(u, v)$.

Since the approximate solutions (and their initial data) have compact support, we can take $\varphi \equiv 1$ in (5.2). Hence

$$
I_{2}(1)+I_{3}(1) \leq \int_{\mathbb{R}} \frac{\left(v^{\Delta x}(x, 0)\right)^{2}}{2} d x-I_{4}(1) \leq C-I_{4}(1),
$$

for a constant $C$ independent of $\Delta x$ (but dependent on $X, T$ ).

Let us estimate $I_{2}$. Here and elsewhere, we write $v_{ \pm}^{n}$ for $v^{\Delta x}\left(\cdot, t^{n} \pm\right)$. Equipped with this notation, we find

$$
\begin{aligned}
I_{2}(\varphi) & =\frac{1}{2} \sum_{n, j} \int_{x_{j-1 / 2}}^{x_{j+1 / 2}}\left(v_{-}^{n}\right)^{2}-\left(v_{j}^{n}\right)^{2} d x \\
& =\frac{1}{2} \sum_{n, j} \int_{x_{j-1 / 2}}^{x_{j+1 / 2}}\left(v_{-}^{n}-v_{j}^{n}\right)^{2} d x-\sum_{n, j} \int_{x_{j-1 / 2}}^{x_{j+1 / 2}} v_{j}^{n}\left(v_{-}^{n}-v_{j}^{n}\right) d x \\
& =\frac{1}{2} \sum_{n, j} \int_{x_{j-1 / 2}}^{x_{j+1 / 2}}\left(v_{-}^{n}-v_{j}^{n}\right)^{2} d x,
\end{aligned}
$$

where the last equality is a consequence of (4.5).

Regarding the term $I_{3}(\varphi)$, we have that $v^{\Delta x}$ is locally the exact solution of a scalar Riemann problem with a "constant coefficient". Thus

$$
\sigma \llbracket \eta\left(v^{\Delta x}, u^{\Delta x}\right) \rrbracket-\llbracket Q\left(v^{\Delta x}, u^{\Delta x}\right) \rrbracket \geq 0,
$$

and consequently $I_{3}(\varphi) \geq 0$.

It remains to estimate $I_{4}$, which is done using Lemma 5.1 as follows:

$$
\left|I_{4}(\varphi)\right| \leq \Delta t \sum_{n, j}\left|Q\left(u_{j-1 / 2}^{n}, v_{j}^{n}\right)-Q\left(u_{j+1 / 2}^{n}, v_{j}^{n}\right)\right| \leq C\left|u_{0}\right|_{B V} T .
$$

Collecting the above bounds the estimates (5.6) and (5.7) follow from (5.8). 
Lemma 5.3 gives a bound on the variation of the approximate solution across the discrete time levels. This estimate can be converted to an estimate on the spatial variation as in [15] (we omit the details).

Lemma 5.4. Let $v_{j}^{n}$ be defined by the SSG-scheme (cf. Subsection 4.1). There exists a constant $C=C(X, T)$ independent of $\Delta x$ such that

$$
\Delta x \sum_{n, j}\left|v_{j+1}^{n}-v_{j}^{n}\right|^{2} \leq C .
$$

We carry on by proving the $W_{\text {loc }}^{-1,2}$ compactness required by Lemma 3.1.

Lemma 5.5. Let $v^{\Delta x}$ be generated by the SSG-scheme (cf. Subsection 4.1). Let $u$ be the unique entropy solution to the first equation in (1.1). Equipped with any scalar entropy/entropy flux pair $(\eta, Q)$, form the distribution

$$
\mu^{\Delta x}:=\eta\left(u, v^{\Delta x}\right)_{t}+Q\left(u, v^{\Delta x}\right)_{x} .
$$

Then $\left\{\mu^{\Delta x}\right\}_{\Delta x>0}$ belongs to a compact subset of $W_{\mathrm{loc}}^{-1,2}\left(\mathbb{R} \times \mathbb{R}^{+}\right)$.

Proof. In what follows we fix a bounded open set $\Omega \subset \mathbb{R} \times \mathbb{R}^{+}$, which we can assume is of the form $(-X, X) \times(0, T)$ with $X>0$ and $T=N \Delta t$ for some integer $N$. We split $\mu^{\Delta x}$ as $\mu_{1}^{\Delta x}+\mu_{2}^{\Delta x}$, where

$$
\begin{aligned}
& \mu_{1}^{\Delta x}:=\left[\eta\left(u, v^{\Delta x}\right)-\eta\left(u^{\Delta x}, v^{\Delta x}\right)\right]_{t}+\left[Q\left(u, v^{\Delta x}\right)-Q\left(u^{\Delta x}, v^{\Delta x}\right)\right]_{x}, \\
& \mu_{2}^{\Delta x}:=\eta\left(u^{\Delta x}, v^{\Delta x}\right)_{t}+Q\left(u^{\Delta x}, v^{\Delta x}\right)_{x} .
\end{aligned}
$$

In view of Lemma 5.1, fixing any $q_{1} \in(1,2]$, the sequence $\left\{\mu_{1}^{\Delta x}\right\}$ is clearly compact in $W^{-1, q_{1}}(\Omega)$. It remains to estimate $\mu_{2}^{\Delta x}$.

Let $\phi \in C_{c}(\Omega)$. Proceeding as in the proof of Lemma 5.3 we find

$$
\left|\iint_{\Omega} \mu_{2}^{\Delta x} \varphi d x\right| \leq\left|I_{2}(\varphi)\right|+\left|I_{3}(\varphi)\right|+\left|I_{4}(\varphi)\right|,
$$

where $I_{2}, I_{3}, I_{4}$ are defined in (5.3), (5.4), (5.5) respectively.

By standard arguments (see [14]) using (5.7) and the spatial $B V$ regularity part of Lemma 5.1,

$$
\left|I_{3}(\varphi)\right|,\left|I_{4}(\varphi)\right| \leq C\|\varphi\|_{L^{\infty}(\Omega)}, \quad \text { for some constant } C \text { independent of } \Delta x,
$$

and hence $I_{3}, I_{4}$ are bounded (independently of $\Delta x$ ) in the space $\mathcal{M}(\Omega)$ of bounded Radon measures on $\Omega$. Recalling that $\mathcal{M}(\Omega)$ is compactly embedded in $W^{-1, p}(\Omega)$ for any $p \in[1,2)$, we conclude that by a fixing any $q_{2} \in(1,2)$ the sequences $\left\{I_{2}\right\}_{\Delta x>0},\left\{I_{4}\right\}_{\Delta x>0}$ are compact in $W^{-1, q_{2}}(\Omega)$.

It remains to estimate the term $I_{2}$, which we decompose as

$$
I_{2}(\varphi)=I_{2,1}(\varphi)+I_{2,2}(\varphi)+I_{2,3}(\varphi)+I_{2,4}(\varphi)
$$

where (we still utilize $v_{-}^{n}$ as short-hand notation for $v^{\Delta x}\left(\cdot, t^{n}-\right)$ )

$$
\begin{aligned}
& I_{2,1}(\varphi)=\sum_{n, j} \int_{x_{j-1 / 2}}^{x_{j}}\left(\eta\left(u_{j-1 / 2}^{n-1}, v_{-}^{n}\right)-\eta\left(u_{j-1 / 2}^{n}, v_{-}^{n}\right)\right) \varphi\left(x, t^{n}\right) d x \\
& I_{2,2}(\varphi)=\sum_{n, j} \int_{x_{j}}^{x_{j+1 / 2}}\left(\eta\left(u_{j+1 / 2}^{n-1}, v_{-}^{n}\right)-\eta\left(u_{j+1 / 2}^{n}, v_{-}^{n}\right)\right) \varphi\left(x, t^{n}\right) d x
\end{aligned}
$$




$$
\begin{aligned}
I_{2,3}(\varphi)=\sum_{n, j} \int_{x_{j-1 / 2}}^{x_{j+1 / 2}}\left(\eta\left(u_{j+1 / 2}^{n}, v_{-}^{n}\right)-\eta\left(u_{j+1 / 2}^{n}, v_{j}^{n}\right)\right) \varphi\left(x, t^{n}\right) d x \\
I_{2,4}(\varphi)=\sum_{n, j} \int_{x_{j-1 / 2}}^{x_{j}}\left[\left(\eta\left(u_{j+1 / 2}^{n}, v_{j}^{n}\right)-\eta\left(u_{j-1 / 2}^{n}, v_{j}^{n}\right)\right)\right. \\
\left.-\left(\eta\left(u_{j+1 / 2}^{n}, v_{-}^{n}\right)-\eta\left(u_{j-1 / 2}^{n}, v_{-}^{n}\right)\right)\right] \varphi\left(x, t^{n}\right) d x
\end{aligned}
$$

By Lemmas 5.1 and 5.2 and

$$
\left|I_{2,1}(\varphi)\right|,\left|I_{2,2}(\varphi)\right|,\left|I_{2,4}(\varphi)\right| \leq C\|\varphi\|_{L^{\infty}(\Omega)},
$$

and accordingly $\left\{I_{2,1}\right\}_{\Delta x>0},\left\{I_{2,2}\right\}_{\Delta x>0},\left\{I_{2,4}\right\}_{\Delta x>0}$ are compact sequences in $W^{-1, q_{2}}(\Omega)$, where $q_{2} \in[1,2)$ has been fixed before.

To continue we write

$$
I_{2,3}(\varphi)=I_{2,3,1}(\varphi)+I_{2,3,2}(\varphi)
$$

where

$$
\begin{aligned}
& I_{2,3,1}(\varphi)=\sum_{n, j} \int_{x_{j-1 / 2}}^{x_{j+1 / 2}}\left(\eta\left(u_{j+1 / 2}^{n}, v_{-}^{n}\right)-\eta\left(u_{j+1 / 2}^{n}, v_{j}^{n}\right)\right) \varphi_{j}^{n} d x, \quad \varphi_{j}^{n}=\varphi\left(x_{j}, t^{n}\right), \\
& I_{2,3,2}(\varphi)=\sum_{n, j} \int_{x_{j-1 / 2}}^{x_{j+1 / 2}}\left(\eta\left(u_{j+1 / 2}^{n}, v_{-}^{n}\right)-\eta\left(u_{j+1 / 2}^{n}, v_{j}^{n}\right)\right)\left(\varphi\left(x, t^{n}\right)-\varphi_{j}^{n}\right) d x .
\end{aligned}
$$

Next

$$
\begin{aligned}
& \eta\left(u_{j+1 / 2}^{n}, v_{-}^{n}\right)-\eta\left(u_{j+1 / 2}^{n}, v_{j}^{n}\right) \\
& \quad=\eta_{v}\left(u_{j+1 / 2}^{n}, v_{j}^{n}\right)\left(v_{-}^{n}-v_{j}^{n}\right)+\frac{1}{2} \eta_{v v}\left(u_{j+1 / 2}^{n}, \theta_{j}^{n}\right)\left(v_{-}^{n}-v_{j}^{n}\right)^{2}
\end{aligned}
$$

for some intermediate value $\theta_{j}^{n}(x)$. Taking into account the definition of $v_{j}^{n}$, see (4.5), and Lemma 5.3 we therefore obtain

$$
\left|I_{2,3,1}(\varphi)\right|=\left|\sum_{n, j} \varphi_{j}^{n} \int_{x_{j-1 / 2}}^{x_{j+1 / 2}} \frac{1}{2} \eta_{v v}\left(u_{j+1 / 2}^{n}, \theta_{j}^{n}\right)\left(v_{-}^{n}-v_{j}^{n}\right)^{2} d x\right| \leq C\|\varphi\|_{L^{\infty}(\Omega)},
$$

where $C$ is independent of $\Delta x$, and so $\left\{I_{2,3,1}\right\}_{\Delta x>0}$ is compact in $W^{-1, q_{2}}(\Omega)$.

To proceed we assume additionally that $\varphi$ is Hölder continuous with some exponent $\alpha \in(1 / 2,1)$. Applying Hölder's inequality and Lemma 5.3 yield

$$
\begin{aligned}
&\left|I_{2,3,2}(\varphi)\right| \leq\left\{\sum_{n, j} \int_{x_{j-1 / 2}}^{x_{j+1 / 2}}\left(\eta\left(u_{j+1 / 2}^{n}, v_{-}^{n}\right)-\eta\left(u_{j+1 / 2}^{n}, v_{j}^{n}\right)\right)^{2} d x\right\}^{\frac{1}{2}} \\
& \times\left\{\sum_{n, j} \int_{x_{j-1 / 2}}^{x_{j+1 / 2}}\left(\varphi\left(x, t^{n}\right)-\varphi_{j}^{n}\right)^{2} d x\right\}^{\frac{1}{2}} \\
& \leq C\|\phi\|_{C^{0, \alpha}(\Omega)}(\Delta x)^{\alpha-\frac{1}{2}} \leq \tilde{C}\|\phi\|_{W^{1, p}(\Omega)}(\Delta x)^{\alpha-\frac{1}{2}},
\end{aligned}
$$

for some constants $C, \tilde{C}$ that are independent of $\Delta x$. To derive the last inequality we used that $W^{1, p}(\Omega) \subset C^{0, \alpha}(\Omega)$ for $p=2 /(1-\alpha)$, where our $\alpha$ lies in $(1 / 2,1)$. Hence $\left\|I_{2,3,2}\right\|_{W^{-1, q_{3}}}$, with $q_{3}=2 /(1+\alpha)$ and $\alpha \in(1,1 / 2)$, tends to zero as $\Delta x \rightarrow 0$. 
Let us now summarize our findings. We have proved that $\left\{\mu^{\Delta x}\right\}_{\Delta x>0}$ is compact in $W^{-1, q}(\Omega)$ for $q=\min \left(q_{1}, q_{2}, q_{3}\right)<2 /(1+\alpha)<2$. Additonally, by the $L^{\infty}$ bounds on $u, u^{\Delta x}, v^{\Delta x}$ the sequence $\left\{\mu^{\Delta x}\right\}_{\Delta x>0}$ is bounded in $W^{-1, r}(\Omega)$ for any $r \in(2, \infty]$. That being the case, an application of Lemma 3.2 concludes the proof.

In view of the compensated compactness theory (Lemma 3.1), the foregoing lemma allows us to prove convergence of the SSG-scheme.

Theorem 5.1. Let $u^{\Delta x}, v^{\Delta x}$ be generated by the SSG-scheme (cf. Subsection 4.1). Then there exist limit functions $u \in L^{\infty}\left(\mathbb{R} \times \mathbb{R}^{+}\right) \cap B V\left(\mathbb{R} \times \mathbb{R}^{+}\right)$and $v \in L^{\infty}\left(\mathbb{R} \times \mathbb{R}^{+}\right)$ such that along a subsequence as $\Delta x \rightarrow 0$

$$
u^{\Delta x} \rightarrow u, v^{\Delta x} \rightarrow v \text { in } L_{\mathrm{loc}}^{p}\left(\mathbb{R} \times \mathbb{R}^{+}\right) \forall p<\infty \text { and a.e. in } \mathbb{R} \times \mathbb{R}^{+},
$$

The limit pair $(u, v)$ constitutes a weak solution of (1.1).

Proof. We refer to Lemma 5.1 for the convergence of $u^{\Delta x}$. The $L_{\text {loc }}^{p}$ convergence of $v^{\Delta x}$ is a direct consequence of Lemmas 5.5 and 3.1.

It remains to show that the limit pair $(u, v)$ is a weak solution. To this end, let $\tilde{v}^{\Delta x}$ be the piecewise constant function defined as

$$
\tilde{v}^{\Delta x}(x, t)=\sum_{n, j} v_{j}^{n} \chi_{j}^{n}(x, t)
$$

We claim that

$$
\lim _{\Delta x \rightarrow 0}\left\|\tilde{v}^{\Delta x}-v\right\|_{L_{\mathrm{loc}}^{2}\left(\mathbb{R} \times \mathbb{R}^{+}\right)}=0 .
$$

For $(x, t) \in\left[x_{j-1 / 2}, x_{j}\right] \times I^{n}$ we have

$$
\left|\tilde{v}^{\Delta x}-v^{\Delta x}\right|=\left|v_{j}^{n}-v^{\Delta x}\right| \leq\left|v_{j}^{n}-v_{j-1}^{n}\right|,
$$

since $v^{\Delta x}$ is the solution of a scalar Riemann problem with left state $v_{j-1}^{n}$ and right state $v_{j}^{n}$. Therefore

$$
\begin{aligned}
\left\|\tilde{v}^{\Delta x}-v^{\Delta x}\right\|_{L^{2} \operatorname{loc}\left(\mathbb{R} \times \mathbb{R}^{+}\right)}^{2} & =\sum_{n, j} \iint_{I_{j-1 / 2}^{n}}\left(\tilde{v}^{\Delta x}-v^{\Delta x}\right)^{2} d x d t \\
& \leq 2 \Delta x \Delta t \sum_{n, j}\left(v_{j}^{n}-v_{j-1}^{n}\right)^{2} \leq C \Delta t,
\end{aligned}
$$

by Lemma 5.4. Hence (5.9) follows.

Pick a test fuction $\varphi$ having compact support in $\mathbb{R} \times[0, T)$ with $N \Delta t=T$ for some integer $N$. Multiplying the scheme (4.6) by $\varphi_{j}^{n}=\varphi\left(x_{j}, t^{n}\right)$ and doing partial summations, we get

$$
\begin{gathered}
0=\underbrace{\Delta x \sum_{j}\left(v^{N} \varphi_{j}^{N-1}-v_{j}^{0} \varphi_{j}^{0}\right)}_{I_{1}}-\underbrace{\Delta x \Delta t \sum_{n, j} v_{j}^{n} \frac{\varphi_{j}^{n}-\varphi_{j}^{n-1}}{\Delta t}}_{I_{2}} \\
-\underbrace{\Delta x \Delta t \sum_{n, j} g^{\mathrm{G}}\left(u_{j-1 / 2}^{n}, v_{j-1}^{n}, v_{j}^{n}\right) \frac{\varphi_{j}^{n}-\varphi_{j-1}^{n}}{\Delta x}}_{I_{3}} .
\end{gathered}
$$


It is straightforward to show that

$$
\lim _{\Delta x \rightarrow 0} I_{1}=-\int_{\mathbb{R}} v_{0} \varphi(x, 0) d x, \quad \lim _{\Delta x \rightarrow 0} I_{2}=\iint_{\mathbb{R} \times \mathbb{R}^{+}} v \varphi_{t} d x d t .
$$

Next we study the term $I_{3}$, which we rewrite as follows:

$$
\begin{aligned}
I_{3}= & \frac{\Delta x \Delta t}{2} \sum_{n, j} g^{\mathrm{G}}\left(u_{j-1 / 2}^{n}, v_{j-1}^{n}, v_{j}^{n}\right) \frac{\varphi_{j}^{n}-\varphi_{j-1}^{n}}{\Delta x} \\
& +\frac{\Delta x \Delta t}{2} \sum_{n, j} g^{\mathrm{G}}\left(u_{j+1 / 2}^{n}, v_{j}^{n}, v_{j+1}^{n}\right) \frac{\varphi_{j+1}^{n}-\varphi_{j}^{n}}{\Delta x} \\
= & \frac{\Delta x \Delta t}{2} \sum_{n, j} g\left(u_{j-1 / 2}^{n}, v_{j}^{n}\right) \frac{\varphi_{j}^{n}-\varphi_{j-1}^{n}}{\Delta x} \\
& +\frac{\Delta x \Delta t}{2} \sum_{n, j} g\left(u_{j+1 / 2}^{n}, v_{j}^{n}\right) \frac{\varphi_{j+1}^{n}-\varphi_{j}^{n}}{\Delta x}+E_{1}^{\Delta x}+E_{2}^{\Delta x} \\
= & \iint_{\mathbb{R} \times \mathbb{R}^{+}} g\left(u^{\Delta x}, \tilde{v}^{\Delta x}\right) \varphi_{x} d x d t+\mathcal{O}(\Delta x)+E_{1}^{\Delta x}+E_{2}^{\Delta x},
\end{aligned}
$$

where

$$
\begin{aligned}
& E_{1}^{\Delta x}=\frac{\Delta x \Delta t}{2} \sum_{n, j}\left[g^{\mathrm{G}}\left(u_{j-1 / 2}^{n}, v_{j-1}^{n}, v_{j}^{n}\right)-g\left(u_{j-1 / 2}^{n}, v_{j}^{n}\right)\right] \frac{\varphi_{j}^{n}-\varphi_{j-1}^{n}}{\Delta x} \\
& E_{2}^{\Delta x}=\frac{\Delta x \Delta t}{2} \sum_{n, j}\left[g^{\mathrm{G}}\left(u_{j+1 / 2}^{n}, v_{j}^{n}, v_{j+1}^{n}\right)-g\left(u_{j+1 / 2}^{n}, v_{j}^{n}\right)\right] \frac{\varphi_{j+1}^{n}-\varphi_{j}^{n}}{\Delta x}
\end{aligned}
$$

By consistency/Lipschitz properties of the Godunov flux, Hölder's inequality, and Lemma 5.4,

$$
\left|E_{1}^{\Delta x}\right|,\left|E_{2}^{\Delta x}\right| \leq C_{\varphi} \sqrt{\Delta x}
$$

where $C_{\varphi}$ depends on $\varphi$ but not $\Delta x$. Hence

$$
\lim _{\Delta x \rightarrow 0} I_{3}=\iint_{\mathbb{R} \times \mathbb{R}^{+}} g(u, v) \varphi_{x} d x d t .
$$

Therefore $v$ is a weak solution of (1.1).

5.2. The ASG-scheme. We begin by pointing out that Lemmas 5.1 and 5.2 for the SSG-scheme continue to hold for the ASG-scheme (with the notation porperly adjusted).

To carry out the convergence analysis for the ASG-scheme we make a digression and present some general results on entropy estimates for conservation laws with discontinuous coefficients. To this end, consider the Riemann problem $[13,16,17]$

$$
\left\{\begin{array}{llll}
v_{t}+g\left(u_{l}, v\right)_{x}=0, & v(x, 0)=v_{l} & x<0 \\
v_{t}+g\left(u_{r}, v\right)_{x}=0, & v(x, 0)=v_{r} & x>0
\end{array}\right.
$$

where $u_{l, r}$ and $v_{l, r}$ are constants. The Rankine-Hugoniot condition states that the values

$$
v_{l, r}^{\prime}=\lim _{x \rightarrow 0-,+} v(x, t)
$$

are such that

$$
g_{0}:=g\left(u_{l}, v_{l}^{\prime}\right)=g\left(u_{r}, v_{r}^{\prime}\right)
$$


In general, this does not determine $v_{l, r}^{\prime}$ uniquely, and we need additional conditions. We choose to use the so called minimal jump entropy condition which states that among the possible choices, we select $v_{l}^{\prime}$ and $v_{r}^{\prime}$ such that $\left|v_{l}^{\prime}-v_{r}^{\prime}\right|$ is minimal. This implies the following

$$
\begin{aligned}
& v_{l}^{\prime} \leq v_{r}^{\prime} \Longrightarrow \begin{cases}g\left(u_{l}, v\right) \geq g\left(u_{l}, v_{l}^{\prime}\right), & \text { for all } v \in\left[v_{l}^{\prime}, v_{r}^{\prime}\right], \text { or } \\
g\left(u_{r}, v\right) \geq g\left(u_{r}, v_{r}^{\prime}\right), & \text { for all } v \in\left[v_{l}^{\prime}, v_{r}^{\prime}\right],\end{cases} \\
& v_{r}^{\prime} \leq v_{l}^{\prime} \Longrightarrow \begin{cases}g\left(u_{l}, v\right) \leq g\left(u_{l}, v_{l}^{\prime}\right), & \text { for all } v \in\left[v_{r}^{\prime}, v_{l}^{\prime}\right], \text { or } \\
g\left(u_{r}, v\right) \leq g\left(u_{r}, v_{r}^{\prime}\right), & \text { for all } v \in\left[v_{r}^{\prime}, v_{l}^{\prime}\right] .\end{cases}
\end{aligned}
$$

Lemma 5.6. If the values $v_{l}^{\prime}$ and $v_{r}^{\prime}$ are chosen according to the minimal jump entropy condition, then, for any constant $c \in\left[s_{0}, S_{0}\right]$,

$$
Q_{r}\left(v_{r}^{\prime}, c\right)-Q_{l}\left(v_{l}^{\prime}, c\right) \leq\left|g\left(u_{r}, c\right)-g\left(u_{l}, c\right)\right|,
$$

where $Q_{l}$ and $Q_{r}$ are the Kružkov entropy fluxes,

$$
\begin{aligned}
& Q_{l}(v, c)=\operatorname{sign}(v-c)\left(g\left(u_{l}, v\right)-g\left(u_{l}, c\right)\right), \\
& Q_{r}(v, c)=\operatorname{sign}(v-c)\left(g\left(u_{r}, v\right)-g\left(u_{r}, c\right)\right) .
\end{aligned}
$$

Proof. If $\operatorname{sign}\left(v_{l}^{\prime}-c\right)=\operatorname{sign}\left(v_{r}^{\prime}-c\right)$ then the right-hand side of (5.12) equals

$$
\begin{aligned}
\operatorname{sign}\left(v_{l}^{\prime}-c\right)\left(g\left(u_{r}, v_{r}^{\prime}\right)-g\left(u_{r}, c\right)-g\left(u_{l}, v_{l}^{\prime}\right)+g\left(u_{l}, c\right)\right) \\
=\operatorname{sign}\left(v_{l}^{\prime}-c\right)\left(g\left(u_{l}, c\right)-g\left(u_{r}, c\right)\right),
\end{aligned}
$$

and the inequality clearly holds. If $v_{l}^{\prime} \leq v_{r}^{\prime}$ then (5.12) reads

$$
2 g_{0}-g\left(u_{l}, c\right)-g\left(u_{r}, c\right) \leq\left|g\left(u_{r}, c\right)-g\left(u_{l}, c\right)\right|
$$

or

$$
\begin{aligned}
2 g_{0}-\max \left\{g\left(u_{l}, c\right), g\left(u_{r}, c\right)\right\} & -\min \left\{g\left(u_{l}, c\right), g\left(u_{r}, c\right)\right\} \\
\leq & \max \left\{g\left(u_{l}, c\right), g\left(u_{r}, c\right)\right\}-\min \left\{g\left(u_{l}, c\right), g\left(u_{r}, c\right)\right\} .
\end{aligned}
$$

In other words (5.12) is the same as

$$
g_{0} \leq \max \left\{g\left(u_{l}, c\right), g\left(u_{r}, c\right)\right\},
$$

and it is immediate that (5.10) implies this. If $v_{r}^{\prime} \leq v_{l}^{\prime}$ then (5.12) reads

$$
g_{0} \geq \min \left\{g\left(u_{l}, c\right), g\left(u_{r}, c\right)\right\},
$$

which is implied by (5.11).

Let $u_{j}^{n}, v_{j}^{n}, v^{\Delta x}(x, t)$ be defined by the ASG-scheme (cf. Subsection 4.2), and set

$$
v_{j+1 / 2}^{n, \pm}=\lim _{x \rightarrow x_{j+1 / 2} \pm} v^{\Delta x}(x, t), \quad t \in I^{n} .
$$

With $Q_{c}(u, v)=\operatorname{sign}(v-c)(g(u, v)-g(u, c))$, Lemma 5.6 implies that the quantity $Q\left(u_{j+1}^{n}, v_{j+1 / 2}^{n,+}\right)-Q\left(u_{j}^{n}, v_{j+1 / 2}^{n,-}\right)$ is bounded above by $\left|u_{j+1}^{n}-u_{j}^{n}\right|$. To establish the $W_{\text {loc }}^{-1,2}$ compactness for the ASG-scheme we need a similar upper bound for any smooth scalar entropy/entropy flux pair $(\eta(u, v), Q(u, v))$. 
Lemma 5.7. Let $\eta(u, v)$ be a smooth function defined on the rectangle $[\alpha, \beta] \times$ $\left[s_{0}, S_{0}\right]$ and define $Q:[\alpha, \beta] \times[s, S] \rightarrow \mathbb{R}$ by $Q_{v}(u, v)=\eta_{v}(u, v) g_{v}(u, v)$. If $\eta_{v v u}$ is uniformly bounded, then for all $j$ and $n$

$$
Q\left(u_{j+1}^{n}, v_{j+1 / 2}^{n,+}\right)-Q\left(u_{j}^{n}, v_{j+1 / 2}^{n,-}\right) \leq C\left|u_{j+1}^{n}-u_{j}^{n}\right|,
$$

for some constant $C$ independent of $\Delta x$ (but dependent on the smoothness of $\eta$ ).

Proof. Set $h=\left(S_{0}-s_{0}\right) / M$ for some positive integer $M$, and let

$$
c_{i}=s_{0}+i h, \quad i=0, \ldots, M .
$$

For $u \in[\alpha, \beta]$ and $v \in\left[s_{0}, S_{0}\right]$, define the function

$$
\eta^{M}(u, v)=\sum_{i=1} k_{i}(u)\left|v-c_{i}\right|+\eta\left(u, s_{0}\right)+\frac{\eta\left(u, S_{0}\right)-\eta\left(u, s_{0}\right)}{S_{0}-s_{0}}\left(v-s_{0}\right),
$$

where

$$
k_{i}(u)=\frac{1}{2 h}\left(\eta\left(u, c_{i+1}\right)-2 \eta\left(u, c_{i}\right)+\eta\left(u, c_{i-1}\right)\right)=\frac{1}{2} h \eta_{v v}\left(u, \theta_{j}\right),
$$

for some $\theta_{j}$ in $\left(c_{i-1}, c_{i+1}\right)$. Since $v \mapsto \eta^{M}(u, v)$ is the piecewise linear interpolation to $v \mapsto \eta(u, v)$ between the points $c_{i}$, we have that $C h \geq\left|\eta^{M}(u, v)-\eta(u, v)\right|$ for $(u, v) \in[\alpha, \beta] \times\left[s_{0}, S_{0}\right]$ and some non negative constant $C$. Next define the function

$$
Q^{M}(u, v)=\sum_{i}^{M} k_{i}(u) Q_{i}(u, v), \quad Q_{i}(u, v)=\operatorname{sign}\left(v-c_{i}\right)\left(g(u, v)-g\left(u, c_{i}\right)\right) .
$$

Now

$$
\begin{aligned}
\eta_{v}^{M}(u, v) & =\sum_{i} k_{i}(u) \operatorname{sign}\left(v-c_{i}\right), \\
Q_{v}^{M}(u, v) & =\sum_{i} k_{i}(u) \operatorname{sign}\left(v-c_{i}\right) g_{v}(u, v),
\end{aligned}
$$

so that $Q_{v}^{M}(u, v)=\eta_{v}^{M}(u, v) g_{v}(u, v)$.

Then we can use Lemma 5.6 to show that

$$
\begin{aligned}
& k_{i}\left(u_{j+1}^{n}\right) Q_{i}\left(u_{j+1}^{n}, v_{j+1 / 2}^{n,+}\right)-k_{i}\left(u_{j}^{n}\right) Q_{i}\left(u_{j}^{n}, v_{j+1 / 2}^{n,-}\right) \\
& \leq\left|k_{i}\left(u_{j+1}^{n}\right)\right|\left|Q_{i}\left(u_{j+1}^{n}, v_{j+1 / 2}^{n,+}\right)-Q_{i}\left(u_{j}^{n}, v_{j+1 / 2}^{n,-}\right)\right| \\
& \quad+\left|Q_{i}\left(u_{j}^{n}, v_{j+1 / 2}^{n,-}\right)\right|\left|k_{i}\left(u_{j+1}^{n}\right)-k_{i}\left(u_{j}^{n}\right)\right| \\
& \quad \leq C_{1} h\left|\eta_{v v}\left(u_{j+1}^{n}, \theta_{i}\right)\right|\left|u_{j+1}^{n}-u_{j-1}^{n}\right|+C_{2} h\left|\eta_{v v u}\left(\omega_{j+1 / 2}^{n}, \theta_{i}\right)\right|\left|u_{j+1}^{n}-u_{j}^{n}\right| \\
& \leq C h\left|u_{j+1}^{n}-u_{j}^{n}\right|,
\end{aligned}
$$

where $\omega_{j+1 / 2}^{n}$ is between $u_{j}^{n}$ and $u_{j+1}^{n}$. From this it follows that

$$
Q^{M}\left(u_{j+1}^{n}, v_{j+1 / 2}^{n,+}\right)-Q^{M}\left(u_{j}^{n}, v_{j+1 / 2}^{n,-}\right) \leq C\left(S_{0}-s_{0}\right)\left|u_{j+1}^{n}-u_{j}^{n}\right| .
$$

Now we can let $M \rightarrow \infty$ and conclude that (5.14) holds.

Now that the preliminaries are out of the way, we set out to prove convergence of the ASG-scheme, following the route laid out for the SSG-scheme. 
Lemma 5.8. Let $v^{\Delta x}$ be defined by the ASG-scheme (cf. Subsection 4.2). There exists a constant $C=C(X, T)$ independent of $\Delta x$ such that

$$
\sum_{j, n} \int_{x_{j-1 / 2}}^{x_{j+1 / 2}}\left|v^{\Delta x}\left(x, t^{n}-\right)-v_{j}^{n}\right|^{2} d x \leq C .
$$

Additionally,

$$
\Delta x \sum_{n, j}\left|v_{j+1}^{n}-v_{j}^{n}\right|^{2} \leq C .
$$

Proof. The proof of this lemma is virtually identical to the proof of Lemma 5.4 (see also Lemma 5.4). Indeed for ASG-scheme we still have the decomposition (5.2) except that the term $I_{4}(\varphi)$ now reads

$$
I_{4}(\varphi)=\sum_{n, j} \int_{I^{n}}\left[Q\left(u_{j}^{n}, v_{j+1 / 2}^{n,-}\right)-Q\left(u_{j+1}^{n}, v_{j+1 / 2}^{n,+}\right)\right] \varphi\left(x_{j+1 / 2}, t\right) d t,
$$

where $v_{j+1 / 2}^{n,-}, v_{j+1 / 2}^{n,+}$ are defined in (5.13). Proceeding as before we choose $\eta(u, v)=$ $v^{2} / 2$ and $\varphi=1$, by that means obtaining (5.8). The only new ingredient compared to the SSG-scheme lies in the treatment of $I_{4}(\varphi)$, which can now only be bounded from below. As it happens, Lemma 5.7 yields

$$
I_{4}(\varphi) \geq-C\left|u_{0}\right|_{B V(\mathbb{R})},
$$

for some constant $C$ independent of $\Delta x$. This concludes the proof.

Next, we prove an analogous version of Lemma 5.5 for the ASG-scheme. We remark that in this case, we have to use some specific properties of the Riemann solution (minimal jump entropy condition) to obtain $W_{\text {loc }}^{-1,2}$ compactness. This is a crucial difference with the compactness proof for the SSG-scheme.

Lemma 5.9. Let $v^{\Delta x}$ be generated by the ASG-scheme (cf. Subsection 4.2). Let $u$ be the unique entropy solution to the first equation in (1.1). For any scalar entropy/entropy flux pairs $(\eta, Q)$, form the distribution

$$
\left.\mu^{\Delta x}:=\eta\left(u, v^{\Delta x}\right)_{t}+Q u, v^{\Delta x}\right)_{x} .
$$

Then $\left\{\mu^{\Delta x}\right\}_{\Delta x>0}$ belongs to a compact subset of $W_{\text {loc }}^{-1,2}\left(\mathbb{R} \times \mathbb{R}^{+}\right)$.

Proof. Since the proof is similar to the proof of Lemma 5.5, we shall only outline the main differences. We using the notation of that proof and additionally write $u_{ \pm}^{n}$ instead of $u^{\Delta x}\left(\cdot, t^{n} \pm\right)$.

For any scalar entropy-entropy flux pair $(\eta, Q)$ we decompose the term $I_{2}$ as

$$
\begin{aligned}
I_{2}(\varphi):= & \sum_{n} \int_{\mathbb{R}}\left(\eta\left(u_{-}^{n}, v_{-}^{n}\right)-\eta\left(u_{+}^{n}, v_{+}^{n}\right)\right) \varphi\left(x, t^{n}\right) d x \\
= & \underbrace{\sum_{n} \int_{\mathbb{R}}\left(\eta\left(u_{-}^{n}, v_{-}^{n}\right)-\eta\left(u_{+}^{n}, v_{-}^{n}\right)\right) \varphi\left(x, t^{n}\right) d x}_{I_{2,1}(\varphi)} \\
& +\underbrace{\sum_{n} \int_{\mathbb{R}}\left(\eta\left(u_{+}^{n}, v_{-}^{n}\right)-\eta\left(u_{+}^{n}, v_{+}^{n}\right)\right) \varphi\left(x, t^{n}\right) d x}_{I_{2,2}(\varphi)} .
\end{aligned}
$$


Now

We continue by splitting $I_{2,2}$ as follows:

$$
\left|I_{2,1}(\varphi)\right| \leq C\|\varphi\|_{L^{\infty}(\Omega)} .
$$

$$
\begin{aligned}
I_{2,2}(\varphi)= & \underbrace{\sum_{n, j} \int_{x_{j-1 / 2}}^{x_{j+1 / 2}}\left(\eta\left(u_{j}^{n}, v_{-}^{n}\right)-\eta\left(u_{j}^{n}, v_{j}^{n}\right)\right) \varphi_{j}^{n} d x}_{I_{2,2,1}(\varphi)} \\
& +\underbrace{\sum_{n, j} \int_{x_{j-1 / 2}}^{x_{j+1 / 2}}\left(\eta\left(u_{j}^{n}, v_{-}^{n}\right)-\eta\left(u_{j}^{n}, v_{j}^{n}\right)\right)\left(\varphi\left(x, t^{n}\right)-\varphi_{j}^{n}\right) d x}_{I_{2,2,2}(\varphi)}
\end{aligned}
$$

where $\varphi_{j}^{n}=\varphi\left(x_{j}, t^{n}\right)$.

As before we write $I_{2,2,1}$ as

$$
I_{2,2,1}(\varphi)=\frac{1}{2} \sum_{n, j} \varphi_{j}^{n} \int_{x_{j-1 / 2}}^{x_{j+1 / 2}} \eta_{v v}\left(u_{j}^{n}, \theta_{j}^{n}\right)\left(v_{-}^{n}-v_{j}^{n}\right) d x,
$$

where $\theta_{j}^{n}(x)$ is some value between $v_{j}^{n}$ and $v_{-}^{n}$. By Lemma 5.8

$$
\left|I_{2,2,1}(\varphi)\right| \leq C\|\varphi\|_{L^{\infty}(\Omega)} .
$$

Arguing as earlier we deduce

$$
\left|I_{2,2,2}(\varphi)\right| \leq C\|\varphi\|_{C^{0, \alpha}(\Omega)}(\Delta x)^{\alpha-1 / 2}, \quad \alpha \in(1 / 2,1) .
$$

Compared with the proof of Lemma 5.5, the key difference lies in the handling of the term $I_{4}(\varphi)$ (defined in (5.16)). We use the specific structure of the Riemann solution to estimate this term.

In what follows we utilize the following notation $\left(v_{j+1 / 2}^{n, \pm}\right.$ are defined in (5.13)):

$$
\llbracket Q \rrbracket_{j+1 / 2}^{n}=Q\left(u_{j}^{n}, v_{j+1 / 2}^{n,-}\right)-Q\left(u_{j+1}^{n}, v_{j+1 / 2}^{n,+}\right) .
$$

We have

$$
\begin{aligned}
\llbracket Q \rrbracket_{j+1 / 2}^{n} & =Q\left(u_{j}^{n}, v_{j+1 / 2}^{n,-}\right)-Q\left(u_{j+1}^{n}, v_{j+1 / 2}^{n,+}\right) \\
& =\underbrace{Q\left(u_{j}^{n}, v_{j+1 / 2}^{n,-}\right)-Q\left(u_{j+1}^{n}, v_{j+1 / 2}^{n,+}\right)}_{\llbracket Q_{1} \rrbracket_{j+1 / 2}^{n}}+\underbrace{Q\left(u_{j+1}^{n}, v_{j+1 / 2}^{n,-}\right)-Q\left(u_{j+1}^{n}, v_{j+1 / 2}^{n,+}\right)}_{\llbracket Q_{2} \rrbracket_{j+1 / 2}^{n}}
\end{aligned}
$$

Since the numerical solutions are bounded, we can produce a constant $C$, independent of $\Delta x$, such that

$$
\left|\llbracket Q_{1} \rrbracket_{j+1 / 2}^{n}\right| \leq C\left|u_{j}^{n}-u_{j+1}^{n}\right|
$$

and

$$
\begin{aligned}
\llbracket Q_{2} \rrbracket_{j+1 / 2}^{n} & =\int_{v_{j+1 / 2}^{n,+}}^{v_{j+1 / 2}^{n,-}} Q_{v}\left(u_{j+1}^{n}, \xi\right) d \xi \\
& =\int_{v_{j+1 / 2}^{n,+}}^{v_{j+1 / 2}^{n,-}} \eta_{v}\left(u_{j+1}, \xi\right) g_{v}\left(u_{j+1}^{n}, \xi\right) d \xi
\end{aligned}
$$


By using the boundedness of the numerical solutions and the smoothness of $\eta$, we get

$$
\left|\llbracket Q_{2} \rrbracket_{j+1 / 2}^{n}\right| \leq C \llbracket Q_{3} \rrbracket_{j+1 / 2}^{n}, \quad \llbracket Q_{3} \rrbracket_{j+1 / 2}^{n}:=\int_{v_{j+1 / 2}^{n,+}}^{v_{j+1 / 2}^{n,-}}\left|g_{v}\left(u_{j+1}^{n}, \xi\right)\right| d \xi .
$$

To estimate $\llbracket Q_{3} \rrbracket_{j+1 / 2}^{n}$, we are going to make a simplifying assumption, namely that $g(u, \cdot)$ is a function having at most one local minimum (respectively maximum) and no local maxima (respectively minima) in $[s, S]$ for all $u \in[\alpha, \beta]$. This assumption is quite general as it includes the fluxes for the triangular three-phase flow model in Section 2. Although the more general case of finitely many points of extrema can also be handled, we will not do so here since it is (only) notationally more cumbersome. In what follows we present the details only for the case of local minima; The case of local maxima follows along the same lines.

For fixed $n$ and $j$, let $\theta_{j}^{n}$ and $\theta_{j+1}^{n}$ denote the local minima of the fluxes $g\left(u_{j}^{n}, \cdot\right)$ and $g\left(u_{j+1}^{n}, \cdot\right)$ respectively. Without loss of generality assume that $\theta_{j+1}^{n} \leq \theta_{j}^{n}$ and $g\left(u_{j}^{n}, \theta_{j}^{n}\right) \leq g\left(u_{j+1}^{n}, \theta_{j+1}^{n}\right)$. The solution of the Riemann problem (4.8) with data $\left(v_{j}^{n}, v_{j+1}^{n}\right)$ can be grouped into the four cases, each of which will be detailed below.

Case $1\left[g_{v}\left(u_{j}^{n}, v_{j+1 / 2}^{n,-}\right) \geq 0\right.$ and $\left.g_{v}\left(u_{j+1}^{n}, v_{j+1 / 2}^{n,+}\right) \geq 0\right]$. In this case we have $v_{j+1 / 2}^{n,-} \geq \theta_{j}^{n} \geq \theta_{j+1}^{n}$ and $v_{j+1 / 2}^{n,+} \geq \theta_{j+1}^{n}$. As $g\left(u_{j}^{n}, v_{j+1 / 2}^{n,-}\right)=g\left(u_{j+1}^{n}, v_{j+1 / 2}^{n,+}\right)$ by the Rankine-Hugoniot condition at the interface $x_{j+1 / 2}$, it follows that

$$
\begin{aligned}
\llbracket Q_{3} \rrbracket_{j+1 / 2}^{n}= & g\left(u_{j+1}^{n}, v_{j+1 / 2}^{n,-}\right)-g\left(u_{j+1}^{n}, v_{j+1 / 2}^{n,+}\right) \\
= & g\left(u_{j+1}^{n}, v_{j+1 / 2}^{n,-}\right)-g\left(u_{j}^{n}, v_{j+1 / 2}^{n,-}\right) \\
& +g\left(u_{j}^{n}, v_{j+1 / 2}^{n,-}\right)-g\left(u_{j+1}^{n}, v_{j+1 / 2}^{n,+}\right) \\
= & g\left(u_{j+1}^{n}, v_{j+1 / 2}^{n,-}\right)-g\left(u_{j}^{n}, v_{j+1 / 2}^{n,-}\right),
\end{aligned}
$$

from which we conclude there is a constant independent of $\Delta x$ such that

$$
\left|\llbracket Q_{3} \rrbracket_{j+1 / 2}^{n}\right| \leq C\left|u_{j}^{n}-u_{j+1}^{n}\right| \text {. }
$$

Case $2\left[g_{v}\left(u_{j}^{n}, v_{j+1 / 2}^{n,-}\right) \leq 0\right.$ and $\left.g_{v}\left(u_{j+1}^{n}, v_{j+1 / 2}^{n,+}\right) \leq 0\right]$. Proceeding as in Case 1, we obtain again (5.17).

Case $3\left[g_{v}\left(u_{j}^{n}, v_{j+1 / 2}^{n,-}\right) \leq 0\right.$ and $\left.g_{v}\left(u_{j+1}^{n}, v_{j+1 / 2}^{n,+}\right) \geq 0\right]$. This is the undercompressive case. In this case, the minimal jump entropy condition [16] implies that either $v_{j+1 / 2}^{n,-}=v_{j+1 / 2}^{n,+}=\alpha_{j, j+1}^{n}$ (if the adjacent fluxes intersect at a point $\alpha_{j, j+1}^{n}$ with $g_{v}\left(u_{j}^{n}, \alpha_{j, j+1}^{n}\right)<0$ and $g_{v}\left(u_{j+1}^{n}, \alpha_{j, j+1}^{n}\right)>0$ ) or $v_{j+1 / 2}^{n,+}=\theta_{j+1}^{n}$ (otherwise). In either case, following exactly the proof of (5.17), we obtain

$$
\left|\llbracket Q_{2,3} \rrbracket_{j+1 / 2}^{n}\right| \leq\left|g\left(u_{j+1}^{n}, v_{j+1 / 2}^{n,-}\right)-g\left(u_{j+1}^{n}, v_{j+1 / 2}^{n,+}\right)\right| \leq C\left|u_{j}^{n}-u_{j+1}^{n}\right| .
$$

Case $4\left[g_{v}\left(u_{j}^{n}, v_{j+1 / 2}^{n,-}\right) \geq 0\right.$ and $\left.g_{v}\left(u_{j+1}^{n}, v_{j+1 / 2}^{n,+}\right) \leq 0\right]$. In this case the Riemann solution is of the form $v_{j+1 / 2}^{n,-}=v_{j}^{n}$ and $v_{j+1 / 2}^{n,+}=v_{j+1}^{n}$ (a steady shock is formed at the interface $\left.x_{j+1 / 2}\right)$. Additionally, there holds $v_{j+1}^{n} \leq \theta_{j+1}^{n} \leq \theta_{j}^{n} \leq v_{j}^{n}$. Therefore

$$
\llbracket Q_{2,3} \rrbracket_{j+1 / 2}^{n}=g\left(u_{j+1}^{n}, v_{j}^{n}\right)-g\left(u_{j+1}^{n}, \theta_{j+1}^{n}\right)+g\left(u_{j+1}^{n}, v_{j+1}^{n}\right)-g\left(u_{j+1}^{n}, \theta_{j+1}^{n}\right) .
$$


Expanding in Taylor series up to second order around $\theta_{j+1}^{n}$, keeping in mind that $g_{v}\left(u_{j+1}^{n}, \theta_{j+1}^{n}\right)=0$ and $v_{j+1}^{n} \leq \theta_{j+1}^{n} \leq v_{j}^{n}$, we extract

$$
\left|\llbracket Q_{2,3} \rrbracket_{j+1 / 2}^{n}\right| \leq C\left[\left(v_{j}^{n}-\theta_{j+1}^{n}\right)^{2}\left(v_{j+1}^{n}-\theta_{j+1}^{n}\right)^{2}\right] \leq C\left(v_{j}^{n}-v_{j+1}^{n}\right)^{2},
$$

where the constant $C$ is independent of $\Delta x$.

Thus we have shown that there is a constant $C$ independent of $\Delta x$ such that

$$
\left|\llbracket Q \rrbracket_{j+1 / 2}^{n}\right| \leq C\left[\left|u_{j}^{n}-u_{j+1}^{n}\right|+\left(v_{j}^{n}-v_{j+1}^{n}\right)^{2}\right],
$$

Summing this bound over $j, n$ yields

$$
\left|I_{4}(\varphi)\right| \leq C \Delta t\|\varphi\|_{L^{\infty}(\Omega)} \sum_{j, n}\left|\llbracket Q_{i} \rrbracket_{j+1 / 2}^{n}\right| \leq C\|\varphi\|_{L^{\infty}(\Omega)}, \quad i=1,2,
$$

where we have exploited the $B V$ regularity of $u_{j}^{n}$ and (5.15) to produce a final constant $C$ that is independent of $\Delta x$.

Now we can finish the proof as we did with Lemma 5.5.

We are now in a position to prove convergence of the ASG-scheme.

Theorem 5.2. Let $u^{\Delta x}, v^{\Delta x}$ be generated by the ASG-scheme (cf. Subsection 4.2). Then there exist limit functions $u \in L^{\infty}\left(\mathbb{R} \times \mathbb{R}^{+}\right) \cap B V\left(\mathbb{R} \times \mathbb{R}^{+}\right)$and $v \in L^{\infty}\left(\mathbb{R} \times \mathbb{R}^{+}\right)$ such that along a subsequence as $\Delta x \rightarrow 0$

$$
u^{\Delta x} \rightarrow u, v^{\Delta x} \rightarrow v \text { in } L_{\mathrm{loc}}^{p}\left(\mathbb{R} \times \mathbb{R}^{+}\right) \forall p<\infty \text { and a.e. in } \mathbb{R} \times \mathbb{R}^{+},
$$

The limit pair $(u, v)$ constitutes a weak solution of (1.1).

Proof. The convergence statement for $u^{\Delta x}$ is clear, while the convergence of $v^{\Delta x}$ is a direct consequence of Lemmas 5.9 and 3.1.

What remains to be shown is that $v$ is a weak solution. Pick a test fuction $\varphi$ having compact support in $\mathbb{R} \times[0, T)$ with $N \Delta t=T$ for some integer $N$, and set

$$
\varphi^{\Delta x}(x, t)=\sum_{j} \varphi\left(x_{j}, t\right) \chi_{I_{j}}(x), \quad v_{ \pm}^{n}(x)=v^{\Delta x}\left(x, t^{n} \pm\right) .
$$

Since $v^{\Delta x}$ is a weak solution in each strip $\mathbb{R} \times I^{n}$, cf. (4.8), we can work out the details as follows:

$$
\begin{aligned}
& \iint_{\mathbb{R} \times \mathbb{R}^{+}} v^{\Delta x} \varphi_{t}+g\left(u^{\Delta x}, v^{\Delta x}\right) \varphi_{x} d x d t+\int_{\mathbb{R}} v^{\Delta x}(x, 0) \varphi(x, 0) d x \\
& =\sum_{n} \int_{\mathbb{R}}\left(v_{-}^{n}-v_{+}^{n}\right) \varphi\left(x, t^{n}\right) d x \\
& =\sum_{n} \int_{\mathbb{R}}\left(v_{-}^{n}-v_{+}^{n}\right)\left(\varphi\left(x, t^{n}\right)-\varphi^{\Delta x}\left(x, t^{n}\right)\right) d x \\
& \leq\left[\sum_{n} \int_{\mathbb{R}}\left(v_{-}^{n}-v_{+}^{n}\right)^{2} d x\right]^{1 / 2}\left[\sum_{n} \int_{\mathbb{R}}\left(\varphi\left(x, t^{n}\right)-\varphi^{\Delta x}\left(x, t^{n}\right)\right)^{2} d x\right]^{1 / 2} \leq C \sqrt{\Delta x},
\end{aligned}
$$

where we have used Hölder's inequality and Lemma 5.8. Since $C$ is independent of $\Delta x$, sending $\Delta x \rightarrow 0$ shows that the limit pair $(u, v)$ is a weak solution of (1.1). 


\section{NUMERICAL EXPERIMENTS}

We have tested the different schemes designed in this paper and described in Section 4 on a wide variety of test problems and have found the results to be in accordance with the theory. We report two experiments here.

6.0.1. Example 1. We start with a simple model problem in order to illustrate the numerical schemes. We choose the following flux functions:

$$
f(u)=\frac{1}{2} u^{2}, \quad g(u, v)=4 u v(v-1),
$$

and Riemann initial data

$$
u(x, 0)=\left\{\begin{array}{ll}
0.75, & x<0, \\
0.25, & x \geq 0,
\end{array} \quad v(x, 0)=0.5 .\right.
$$

For this problem we have an explicit form of the exact solution given by

$$
u(x, t)=\left\{\begin{array}{ll}
3 / 4, & x<t / 2, \\
1 / 4, & x \geq t / 2,
\end{array} \quad v(x, t)= \begin{cases}1 / 2, & x<-t, \\
5 / 6, & -t \leq x<t / 2, \\
1 / 2, & x \geq t / 2 .\end{cases}\right.
$$

In Figure 1 we show the approximations for $v$ at $t=0.75$ using the SSG- and ASG-schemes with $\Delta x=1 / 20$ in the interval $[-1,1]$. From this it seems that the both schemes perform equally well, and this impression is confirmed by other computations. As expected, the schemes have some numerical diffusion at the shocks yet the shocks speeds are captured accurately. Since we have a formula for

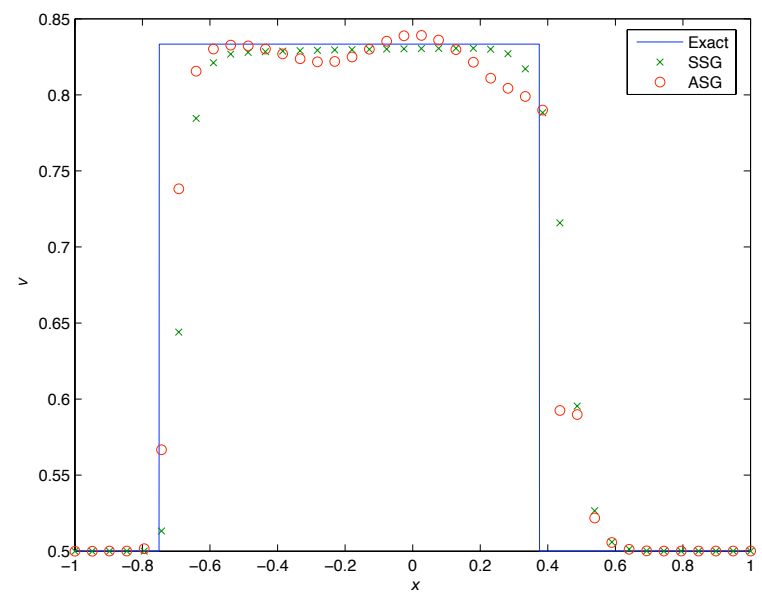

Figure 1. Example 1, the SSG- and the ASG-scheme with $\Delta x=$ $1 / 20$ and $t=0.75$.

the exact solution in this case, we have computed the relative errors in the $L^{1}$ norm for various $\Delta x$. The relative errors are defined as

$$
e=\frac{\sum_{j}\left|v^{\Delta x}\left(x_{j}, t\right)-v_{\mathrm{ex}}\left(x_{j}, t\right)\right|}{\sum_{j}\left|v_{\mathrm{ex}}\left(x_{j}, t\right)\right|}
$$


where $v_{\text {ex }}$ denotes the exact solution, and $t=0.75$. These errors are reported in Table 1. From this table it seems that both schemes are first order convergent. We

\begin{tabular}{c|ccccccc}
$n$ & 3 & 4 & 5 & 6 & 7 & 8 & 9 \\
\hline SSG & 8.6 & 5.1 & 2.8 & 1.4 & 0.7 & 0.4 & 0.018 \\
ASG & 7.6 & 3.7 & 2.1 & 1.1 & 0.6 & 0.3 & 0.015
\end{tabular}

TABLE 1. $100 \times$ Relative $L^{1}$ error for the SSG- and ASG-scheme s. We used $\Delta x=2^{-n}$ in the interval $[-1,1]$.

have tested both the schemes on several such model problems and obtain similar results.

6.0.2. Example 2. To test the applicability of the triangular model as a model of three-phase flow in porous media, we have compared the results obtained by the triangular and also the full model on a water flooding problem. This also serves as a good test case for the efficiency of the schemes designed in this paper. We use the relative permeabilities

$$
\lambda_{g, w, o}=\frac{1}{\nu_{g, w, o}} S_{g, w, o}^{2}
$$

with $S_{i}$ denoting the saturation of phase $i$, and $\nu_{i}$ the viscosity. We have used the following viscosities

$$
\nu_{g}=1, \quad \nu_{w}=80, \quad \nu_{o}=100
$$

In addition we have set

$$
\rho_{g}=1 / 20, \quad \rho_{w}=1, \quad \rho_{o}=9 / 10,
$$

and have set the gravitational constant and the absolute permeability to unity. This gives the flux functions

$$
\begin{aligned}
& F_{g}(u, v)=\frac{u^{2}}{u^{2}+v^{2} / 100+(1-v-u)^{2} / 80}\left(1-\frac{17 v^{2}}{200}-\frac{19(1-v-u)^{2}}{160}\right), \\
& F_{o}(u, v)=\frac{v^{2}}{100 u^{2}+v^{2}+(5 / 4)(1-v-u)^{2}}\left(1+\frac{v^{2}}{10}+\frac{19(1-v-u)^{2}}{20}\right),
\end{aligned}
$$

where we have set $u=S_{g}$ and $v=S_{o}$. This is the "full" three-phase flow model, and we see that $F_{g}$ is not very dependent on $v$. To define a triangular method we set

$$
v=\frac{1-u}{2} \text { and thus }(1-u-v)=\frac{1-u}{2},
$$

which gives the flux function

$$
F_{g}(u)=\frac{u^{2}}{u^{2}+(9 / 1600)\left(1-u^{2}\right)}\left(1-\frac{163}{3200}(1-u)^{2}\right) .
$$

We have used the initial values

$$
v(x, 0)=\left\{\begin{array}{ll}
0 & x<0, \\
\frac{1}{2}+\frac{1}{4} \sin (2 \pi x) & x \geq 0,
\end{array} \quad u(x, 0)= \begin{cases}0 & x<0 \\
1-v(x, 0) & x>0 .\end{cases}\right.
$$

This is meant to model the situation where one has a mixture of oil and gas in the reservoir, and one attempts to inject water in order to force out the oil and the gas. We have used (6.2) and (6.1) as $f$ and $g$ respectively and the ASG-scheme (which in 
this case coincides with the upwind scheme) to calculate approximate solutions. In Figure 2 we show contour plots of the gas, oil, and water saturations as functions of $x$ and $t$ for $-0.05 \leq x \leq 5$ and $0 \leq t \leq 2$. Again the shocks are captured with some diffusion and other waves are resolved quite well. These results were very similar to results obtained with the full model, although a more thorough justification for using the triangular model is beyond the scope of this paper.
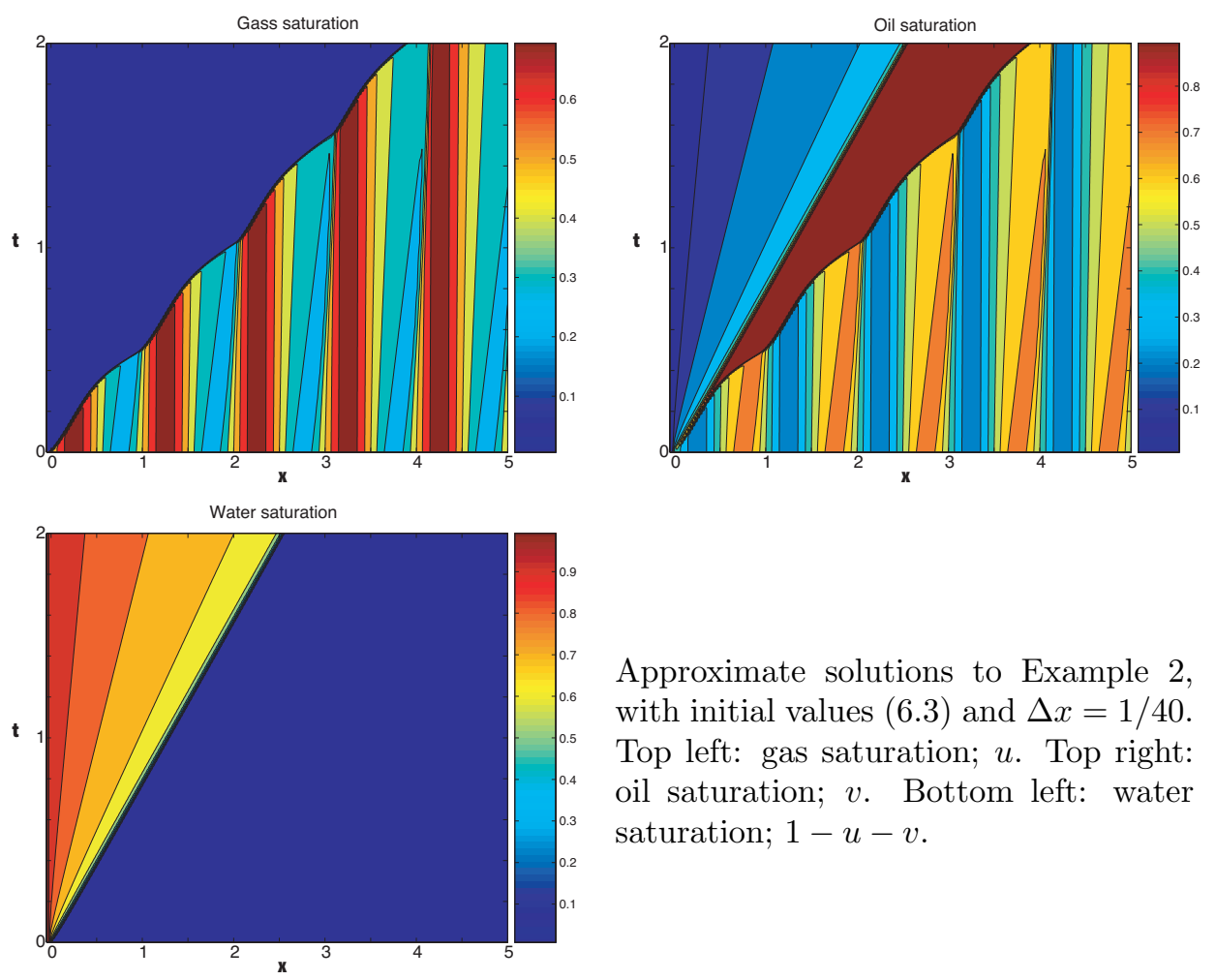

Approximate solutions to Example 2, with initial values $(6.3)$ and $\Delta x=1 / 40$. Top left: gas saturation; $u$. Top right: oil saturation; $v$. Bottom left: water saturation; $1-u-v$.

FiguRE 2

\section{REFERENCES}

[1] Adimurthi and G. D. V. Gowda. Conservation law with discontinuous flux. J. Math. Kyoto Univ., 43(1):27-70, 2003.

[2] Adimurthi, J. Jaffré, and G. D. Veerappa Gowda. Godunov-type methods for conservation laws with a flux function discontinuous in space. SIAM J. Numer. Anal., 42(1):179-208, 2004.

[3] Adimurthi, Siddhartha Mishra and G.D.Veerappa Gowda. Optimal entropy solutions for conservation laws with discontinuous flux. Journal of Hyp. Diff. Eqns., 2(4): 1-56, 2005.

[4] Adimurthi, Siddhartha Mishra and G.D.Veerappa Gowda. Conservation laws with flux function discontinuous in the space variable - III, The general case. Preprint, 2005.

[5] R. Bürger and K. H. Karlsen. On a diffusively corrected kinematic-wave traffic flow model with changing road surface conditions. Math. Models Methods Appl. Sci., 13(12):1767-1799, 2003.

[6] R.Bürger, K.H.Karlsen, N.H.Risebro and J.D.Towers. Well-posedness in $B V_{t}$ and convergence of a difference scheme for continuous sedimentation in ideal clarifier thickener units. Numer. Math., 97 (1):25-65, 2004 
[7] R. Bürger, K. H. Karlsen, E. M. Tory, and W. L. Wendland. Model equations and instability regions for the sedimentation of polydisperse suspensions of spheres. ZAMM Z. Angew. Math. Mech., 82(10):699-722, 2002.

[8] R. Bürger, K. H. Karlsen, and J. D. Towers. A model of continuous sedimentation of flocculated suspensions in clarifier-thickener units. SIAM J. Appl. Math., 65(3):882-940 (electronic), 2005.

[9] G. Chavent and J.Jaffre. Mathematical models and Finite elements for Reservoir simulation. North Holland, Amsterdam, 1986.

[10] G - Q. Chen. Compactness methods and nonlinear hyperbolic conservation laws. Some current topics on nonlinear conservation laws, 33 - 75, Amer. Math. Soc, Providence, R.I, 2000.

[11] G -Q. Chen and D. Wang. The Cauchy problem for the Euler equations for compressible fluids. Handbook of Mathematical Fluid Dynamics, vol 1, Elsevier, 2002.

[12] B. Cockburn, C. Johnson, C.-W. Shu, and E. Tadmor. Advanced numerical approximation of nonlinear hyperbolic equations, volume 1697 of Lecture Notes in Mathematics. SpringerVerlag, Berlin, 1998.

[13] S. Diehl. A conservation law with point source and discontinuous flux function modeling continuous sedimentation. SIAM J. Appl. Math., 56 (2):1980-2007, 1995.

[14] R.J. DiPerna. Convergence of approximate solutions to conservation laws. Arch. Rat. Mech. Anal, 88: 22-70, 1983.

[15] X. Ding, G-Q. Chen and P. Luo. Convergence of the Fractional steps Lax-Friedrichs scheme and Godunov scheme for the isentropic system of gas dynamics. Comm. Math. Phys, 121:6384, 1989 .

[16] T. Gimse and N. H. Risebro. Riemann problems with a discontinuous flux function. In Proc. 3rd Internat. Conf. Hyperbolic Problems, pages 488-502, Uppsala, 1991. Studentlitteratur.

[17] T.Gimse and N.H.Risebro. Solution of Cauchy problem for a conservation law with discontinuous flux function. SIAM J. Math. Anal, 23 (3): 635-648, 1992.

[18] S. Godunov. Finite difference methods for numerical computation of discontinuous solutions of the equations of fluid dynamics. Math.Sbornik, 47: 271-306, 1959.

[19] H. Holden and N. H. Risebro. Front tracking for hyperbolic conservation laws, volume 152 of Applied Mathematical Sciences. Springer-Verlag, New York, 2002.

[20] K. H. Karlsen, K.-A. Lie, J. R. Natvig, H. F. Nordhaug, and H. K. Dahle. Operator splitting methods for systems of convection-diffusion equations: nonlinear error mechanisms and correction strategies. J. Comput. Phys., 173(2):636-663, 2001.

[21] K. H. Karlsen, N.H.Risebro and J.D.Towers. Upwind difference approximations for degenerate parabolic convection-diffusion equations with a discontinuous coefficient. IMA J. Numer. Anal., 22(4):623-664, 2003.

[22] K. H. Karlsen, N.H.Risebro and J.D.Towers. $L^{1}$ stability for entropy solution of nonlinear degenerate parabolic convection-diffusion equations with discontinuous coefficients. Skr. K. Nor. Vidensk. Selsk. ,3, 2003, 49 pages.

[23] K. H. Karlsen and J .D.Towers. Convergence of the Lax-Friedrichs scheme and stability of conservation laws with a discontinuous time-dependent flux. Chinese Ann.Math.Ser B., 25: 287-318, 2004.

[24] C.Klingenberg and N .H.Risebro. Convex conservation laws with discontinuous coefficients: Existence, uniqueness and Asymptotic behavior. Comm. Part. Diff. Eqns 20 (11-12): 1959 $-1990,1995$.

[25] Y. Lu. Hyperbolic conservation laws and the compensated compactness method. Surveys in Pure and Applied Mathematics, Chapman and Hall, CRC, Boca Raton, Fl. 2003.

[26] S. Mishra. Convergence of upwind finite difference schemes for a scalar conservation law with indefinite discontinuities in the flux function. SIAM Jl. Num. Anal, 43(2): 559- 577, 2005.

[27] S. Mishra. Analysis and Numerical approximation of conservation laws with discontinuous coefficients PhD Thesis, Indian Institute of Science, Bangalore, 2005.

[28] D. N. Ostrov. Solutions of Hamilton-Jacobi equations and scalar conservation laws with discontinuous space-time dependence. J. Differential Equations, 182(1):51-77, 2002.

[29] N. Seguin and J. Vovelle. Analysis and approximation of a scalar conservation law with a flux function with discontinuous coefficients. Math. Models Methods Appl. Sci., 13(2):221-257, 2003. 
[30] L. Tartar. Compensated compactness and applications to partial differential equations. In Nonlinear analysis and mechanics: Heriot-Watt symposium, vol IV, pages 136 -212, Pitman, Boston, 1979.

[31] L. Tartar. The compensated compactness method applied to systems of conservation laws. In Systems of Nonlinear partial differential equations, Oxford (1982) volume 111 of NATO Adv. sci. inst. ser. C Math.

[32] J.D.Towers. Convergence of a difference scheme for conservation laws with a discontinuous flux. SIAM J. Numer. Anal.,38(2):681-698, 2000.

[33] J.D. Towers. A difference scheme for conservation laws with a discontinuous flux-the nonconvex case. SIAM J. Numer. Anal.,39(4): 1197-1218, 2001.

(Kenneth Hvistendahl Karlsen)

Centre of Mathematics for Applications (CMA)

UNIVERSITY OF OSLO

P.O. Box 1053, BLindern

N-0316 Oslo, Norway

E-mail address: kennethk@math.uio.no

URL: http://www.math.uio.no/ kennethk/

(Siddhartha Mishra)

Centre of Mathematics for Applications (CMA)

UNIVERSITY OF OSLO

P.O. Box 1053, BLINDERN

$\mathrm{N}-0316$ OsLo, Norway

E-mail address: siddharm@cma.uio.no

(Nils Henrik Risebro)

Centre of Mathematics for Applications (CMA)

UNIVERSITY OF OSLO

P.O. Box 1053, Blindern

N-0316 Oslo, Norway

E-mail address: nilshr@math.uio.no 